Research Article

\title{
Computational Analysis for Rainfall Characterization and Drought Vulnerability in Peninsular India
}

\author{
M. P. Akhtar $\mathbb{D}^{1},{ }^{1}$ Firoz Alam Faroque $\mathbb{D}^{1},{ }^{1}$ L. B. Roy $\mathbb{D}^{2},{ }^{2}$ Mohd. Rizwanullah $\left(\mathbb{D},{ }^{3}\right.$ \\ and Mukesh Didwania $\mathbb{D}^{4}$ \\ ${ }^{1}$ Department of Civil Engineering, School of Civil \& Chemical Engineering (SCCE), Manipal University Jaipur, \\ Rajasthan 303007, India \\ ${ }^{2}$ Department of Civil Engineering, National Institute of Technology, Patna 800015, India \\ ${ }^{3}$ Department of Mathematics \& Statistics, Manipal University Jaipur, Rajasthan 303007, India \\ ${ }^{4}$ Mechanical Engineering Department, Adama Science and Technology University, P.O. Box 1888, Adama, Ethiopia
}

Correspondence should be addressed to Mukesh Didwania; mukeshdidwania4u@gmail.com

Received 14 February 2021; Revised 20 May 2021; Accepted 15 June 2021; Published 30 June 2021

Academic Editor: Hassène Gritli

Copyright $\odot 2021$ M. P. Akhtar et al. This is an open access article distributed under the Creative Commons Attribution License, which permits unrestricted use, distribution, and reproduction in any medium, provided the original work is properly cited.

\begin{abstract}
This paper analyzes the historical rainfall characterization and drought conditions in two major southern states of India, namely, Tamil Nadu and Karnataka, through estimation of meteorological drought indices, namely, drought index (DI), Palmer drought index (PDI), and standardized precipitation index (SPI). Monthly and yearly rainfall data, including temperature, have been considered for 110 years. Deficient rainfall conditions have been identified and compared using annual rainfall classification thresholds. Annual rainfall variability and trend have been estimated using Mann-Kendall test and Sen's slope test. Comparative study on results implies that drought characterization using SPI may amply facilitate the standardization of threshold classification for severity and frequency. Based on threshold classification, it is found that Tamil Nadu witnessed on an average 11 years of moderate drought, 4.36 years of severe drought, and 1.32 years of extreme drought conditions with standard deviations of $4.28,1.87$, and 1.63 years, respectively, during the study period, whereas Karnataka witnessed on an average 9.74 years of moderate drought, 3.91 years of severe drought, and 2.30 years of extreme drought conditions with standard deviations of 4.54, 2.04, and 2.21 years, respectively, during the study period. According to the analysis, drought vulnerability in Tamil Nadu was higher than in Karnataka, based on the number of dry and wet years in terms of SPI threshold values and area covered over 110 years. Karnataka was more susceptible in terms of severity. When compared with other indices, analysis based on drought indices indicates that a single variable-based estimation using SPI is easy to assess and may be significant and definitive in terms of decision making for prioritizing drought mitigation measures in the study area in case of inadequate available data for multiple variable-based drought analysis.
\end{abstract}

\section{Introduction}

Drought is one of the most severe natural hazards, as it brings with it a slew of unforeseen calamities for humans and their societies. Meteorological drought in different regions may have different intensities based on their precipitation pattern. Droughts have the potential to negatively impact agriculture and food security, hydropower generation and industry, human and animal health, livelihood security, personal security, and educational access [1]. Drought may be considered one of major reason for desertification.
Rainfall is one of the most important climatic indicators that determines different drought condition depending upon its long-term intensities, distribution, and frequency [2]. A basic understanding of aridity and drought and their distinction is to be understood well before drought analysis. In meteorology and climatology, aridity is characterised as the degree to which a climate lacks reliable, life-promoting moisture, whereas drought is described as a prolonged period of abnormally dry weather that causes a significant hydrological imbalance. The long-term average water availability, usually precipitation, is compared with the 
long-term average water demand in terms of evapotranspiration to assess aridity. When demand exceeds supply on average, the environment is characterized as arid. However, drought is described as a change in moisture balance that occurs on a monthly (or more frequent) basis. If the water supply for a given month is less than the demand, that month is abnormally dry, and if there is a significant hydrological effect, that month is experiencing a drought. Drought is a temporary condition, whereas aridity is permanent [3].

Several attempts have been made by different researchers to define drought precisely; however, researchers yet to have a universally accepted definition of drought. For regions with varying climatic variability, numerous studies have been carried out and recorded for the study of rainfall trends and drought indices using various methodologies. De et al. [4] provided a credible and concise overview of India's severe weather incidents over the last 100 years (1991-2004). Smarkhtin and Hughes [5] worked out methodology to evolve automated estimation and analyses of meteorological drought characteristics from monthly rainfall data. Severe drought conditions were also included under extreme condition for analysing socioeconomic impact on human society. Pai et al. [6] compiled district-wide drought climatology of the southwest monsoon season over India based on drought indicator estimation, namely, standardized precipitation index (SPI). Yosuf et al. [7] attempted characterisation of drought properties with bivariate copula analysis. Shah et al. [8] reported drought index computation using standard precipitation index method for Surat District in Gujrat (India). Wichitarapongsakum et al. [9] investigated three forecasting models based on the time series technique to predict rainfall using data collected from five rain gauge stations in Thailand's Sakae Krang River basin and computed SPI values to gain a better insight of drought intensity in the region. Langat et al. [10] used Mann-Kandell nonparametric experiments to analyse temporal variabilities, rainfall trend, and discharges in the Tana River Basin in Kenya. Several analytical works related to trend analysis for climatic parameters of regional hydrological time series were presented in the past [11-15]. More recently, Perera and Rathnayake [16] investigated climatic prediction for Colombo, Sri Lanka, using ANN models and discovered that the atmospheric temperature in Colombo can be viewed in relation to other climatic variables. The rainfall, however, does not seem to have a stronger relationship with the other climatic variables. More recently, intensive data based on mathematical analysis with smart computing are highly in practice for various domains apart from drought prediction, such as assessing Covid-19 outbreak effect on urban health and environment [17], neuro fuzzy application in benzene detection and monitoring as air polluting agent and exposure extend to humans [18-22].

Similarly, drought condition and its prediction for arid and semiarid regions across the globe are consistently being investigated to develop efficient decision support systems for regional ground and surface water management and planning. Palmer [23] evolved index to identify drought condition with a statistical correlation with mean and standard deviation of mean. Gibbs and Mahar [24] worked out on rainfall decile as drought indicator. Subsequently, McKee et al. [25] devised more meaningful indicator, namely, standard precipitation indicator, to analyse and prediction regional drought conditions. Many works have been reported [9, 22, 26-28]. Kumar et al. [29] looked at regional drought characteristics, such as frequency, intensity, and occurrence of meteorological droughts in arid and semiarid regions of north-western India and noted region-specific event years with severe drought severity. The results of Mallya et al. [30] provided an insight for a thorough investigation of drought severity and recurrence with predicted drought indices in peninsular India. Such studies need to be considered to get more meaningful results for planning regional drought mitigation measures. Drought characterization, trend assessment, and copulabased bivariate analysis for the central Brahmaputra Valley in Assam are recently presented [31], and drought risk was estimated using joint probabilities and the study area's return period. Many works have been reported on drought analysis with regions with climatic variability [26-28, 31-34]. Al Adaileh et al. [27] attempted evolvement of a drought adaption management system for ground water resources based on combined drought index and vulnerability analysis.

Tamil Nadu and Karnataka situated in the south part of India are water stressed regions in Peninsular India. Tamil $\mathrm{Nadu}$ is India's eleventh largest state, while Karnataka has India's second largest rain-fed city, after Rajasthan. In the state of Karnataka, only 35 to 45 percent of rainwater is currently used to cultivate dryland crops. As a result, there is a lot of potential for improving rainwater harvesting and productive use of it for rain-fed crops to combat drought in such arid regions, provided that an effective study of the intensity and recurrence of drought with spatial distribution is done. It could be accomplished through the estimation and analysis of relevant meteorological drought indicators, as well as statistical analysis of long-term rainfall data.

Furthermore, both regions of Indian peninsula were chosen for comparative analysis of drought situations keeping in view of the prevailing water crisis between them. Tamil Nadu is facing acute water shortage due to erratic rainfall and depleting reservoir levels. The main source of water is Cauvery River Basin, which lies mostly in Karnataka region. Both states have been locked in a long dispute over sharing of Cauvery Water. The conflict has continued since long and impasse persisted $[35,36]$. A lack of multilevel, intersectoral, and participatory approaches has resulted in insufficient water conservation and water usage in both states [36]. This study will facilitate a better scientific insight into prevailing issue with respect to meteorological drought situation and relative regional water scarcity for comparison and help decision makers reach a consensus solution that may lead to equitable share of Kaveri Water for both states.

Considering the previously mentioned, the following are the objectives of this study:

(a) To examine the monotonic pattern of rainfall and compare it for Tamil Nadu and Karnataka regions over a period of 110 years using nonparametric 
methods, such as Mann-Kendall and Sen's slope analysis,

(b) To analyse the annual series of the available historical climatic data and assessment of severity distribution with comparison in both states using estimated drought indices, namely, drought index (DI), Palmer drought index (PDI) and standard precipitation index (SPI), and development of vulnerability map based on a chosen drought indicator.

To begin, this paper reviews the parameters and methodologies that have been used and implemented for evaluating intensity and trend analysis of climate parameters.

\section{Material and Methodology}

2.1. Trend Analysis of Annual Rainfall by Using Mann-Kendall Test [37, 38]. The data point $x_{i}$ of a time series can be assumed to follow the model as follows, and the Mann-Kendall test is used [37, 38]:

$$
x=f(t)+\varepsilon_{i},
$$

where $f(t)$ is a continuous monotonic increasing or decreasing function of time, and the residuals $\varepsilon_{i}$ are supposed to come from the same zero-mean distribution. As a result, the variance of the distribution is believed to be stable over time. The following formula is used to calculate the Man$\mathrm{n}$-Kendall test statistic $\mathrm{S}$ :

$$
S=\sum_{k=1}^{n-1} \sum_{j=k+1}\left(x_{i}-x_{k}\right) n \sum_{i} \operatorname{sgn}\left(x_{i}-x_{k}\right),
$$

where $x_{j}$ and $x_{k}$ are annual quantities in years $j$ and $k$, respectively, and $j>k$, sgn $\left(x_{i}-x_{k}\right)$ can be written as follows:

$$
\operatorname{sgn}\left(x_{j}-x_{k}\right)= \begin{cases}1, & \text { if } x_{j}-x_{k}>0, \\ 0, & \text { if } x_{j}-x_{k}=0, \\ -1, & \text { if } x_{j}-x_{k}<0 .\end{cases}
$$

If $n=9$, the absolute value of $S$ is explicitly compared with Mann and Kendall's theoretical distribution of $S$ [39]. $S$ with a positive or negative meaning indicates an upward or downward trend. The minimum values of $n$, for which these four significance thresholds can be achieved, can be found in the probability table (Table 1 ) for $S$.

The normal approximation test is used if $n \geq 10$. If the number of data values is close to 10 , the validity of the normal approximation may be compromised if there are many equivalent values in the time sequence. To begin, the variance of $S$ is estimated using the following equation, which allows "equals" into account:

$$
\operatorname{VAR}(S)=\frac{1}{18}\left[n(n-1)(2 n+5)-\sum_{p=1}^{q} t_{p}\left(t_{p}-1\right)\left(2 t_{p}+5\right)\right] \text {. }
$$

TABle 1: Significance level verses required $n$.

\begin{tabular}{lc}
\hline Significance level $\alpha$ & Required $\mathrm{n}$ \\
\hline 0.1 & $\geq 4$ \\
0.05 & $\geq 5$ \\
0.01 & $\geq 6$ \\
0.001 & $\geq 7$ \\
\hline
\end{tabular}

The number of equal-valued groups is $q$, and the number of data values in the $p^{\text {th }}$ group is $t_{p}$. The test statistic $Z$ is computed using the values of $S$ and $\operatorname{VAR}(S)$ as follows:

$$
Z= \begin{cases}\frac{S-1}{\sqrt{\mathrm{VAR}},} & \text { if } S>1, \\ 0, & \text { if } S=1, \\ \frac{S+1}{\sqrt{\mathrm{VAR}},} & \text { if } S<1 .\end{cases}
$$

The $Z$ value is used to assess the existence of a statistically significant trend. An upward or downward trend is demonstrated by a positive or negative value of $Z$. The statistic $Z$ has a normal distribution. $H_{0}$ is rejected if the absolute value of $Z$ is greater than $Z_{1-\alpha / 2}$, where $Z_{1-\alpha / 2}$ is derived from the standard normal cumulative distribution tables, to search for an upward or downward monotone trend (a two-tailed test) at the level of significance. The significance levels that were measured were $0.001,0.01,0.05$, and 0.1 .

Despite of the advantage, the influence of serial correlation in the time series on the results of MK test has been found in the literature [13]. The existence of seasonality trends and the presence of autocorrelation are two major issues that occur using the MK test. If the data contains seasonal trends, the modified MK test should be used instead of the original MK test $[11,40]$, and the modified MK test as suggested in Hamed and Ramachandra Rao [41] should be used if the time series autocorrelation is relevant [42].

2.1.1. Sen's Slope Test. To estimate the true slope of an existing trend (as a change per year), the Sen's nonparametric method [43] is used. Sen's nonparametric [43] is used to measure the true slope of a current trend (as a transition every year) in situations, where the pattern may be linear. This means that, in the previous equation, $f(t)$ is as follows:

$$
f(t)=Q_{t}+B,
$$

where $Q$ is the slope and $B$ is a constant. To get the slope estimate $Q$ in the previously mentioned equation, one can calculate the slopes of all data value pairs as shown in the following:

$$
Q_{j}=\frac{x_{j}-x_{k}}{j-k}, \quad j>k
$$

There are as many $N=n(n-1) / 2$ slope estimates $Q_{i}$ as there are $n$ values $x_{j}$ in the time series. The median of these $N$ $Q_{i}$ values is Sen's slope estimator. Sen's estimator is shown as 
follows, and the $N$ values of $Q_{i}$ are ranked from smallest to highest:

$$
\begin{aligned}
& Q=Q_{(N+1) / 2}, \quad \text { if } N \text { is odd }, \\
& Q=\frac{1}{2}\left(Q_{(N / 2)}\right), \quad \text { if } N \text { is even. }
\end{aligned}
$$

2.2. Drought Index. Drought events are computed using a variety of methods in terms of intensity and frequency. The drought index can be calculated using the following [44]:

$$
\text { DI }=\frac{P-X}{s} \times 100,
$$

where the annual precipitation is $P$, the long-term mean is $X$, and the standard deviation is $s$. As shown in Table 2, the DI values can be classified into four categories: no drought, mild drought, moderate drought, and extreme drought.

2.3. Palmer Drought Index. Palmer drought index (PDI), introduced by Palmer [23] to simulate moisture content month by month and compare monthly anomalies at regions with different climatic conditions and seasons, is one of the most common indicators to assess drought. PDI measures the severity of a wet or dry spell; the higher the absolute value, the worse the dry or wet spell. The technique used in this paper is based on Gutmann [45] and Thornthwaite [46].

The PDI was designed to quantify meteorological drought. Rather than being an indication of the impact of a long-term weather phenomenon, it is a prolonged and irregular moisture deficit.

The index is computed by carrying out monthly hydrological accounting for prolonged time series. Then, the coefficients depending on the climatic condition of the study area were obtained. The monthly deviations from normal conditions are converted to measure the amount of moisture required for normal weather each month. Climatically, appropriate precipitation $P_{c}$ for individual time scale (month) is

$$
P_{c}=\alpha P_{\mathrm{et}}+\beta P_{r}+\gamma P_{r o}-\delta P_{l},
$$

where $P_{\text {et }}$ is the potential evapotranspiration, $P_{r}$ is the potential recharge, $P_{r o}$ is the potential runoff, $P_{1}$ is the potential loss, and coefficients $\alpha, \beta, \gamma, \delta$ are the ratios of long-term mean quantities for evapotranspiration, recharge to potential recharge, runoff to potential runoff, and loss to potential loss, respectively. $P_{\text {et }}$ is computed using simplified Thornthwaite process [46]. Potential recharge, potential loss, and potential runoff can be computed using following equations [47]:

$$
\begin{aligned}
& P_{r}=\mathrm{AWC}-\left(S_{s}+S_{u}\right), \\
& P_{l}=P_{\mathrm{ls}}+P_{\mathrm{lu}},
\end{aligned}
$$

$$
\begin{aligned}
& P_{\mathrm{ls}}=\min \left(P_{\mathrm{et}}, S_{s}\right), \\
& P_{\mathrm{lu}}=\frac{\left(P_{\mathrm{et}}-P_{\mathrm{ls}}\right) S_{u}}{\mathrm{AWC}}, \quad P_{\mathrm{lu}} \leq S_{u} .
\end{aligned}
$$

Potential runoff $\left(P_{\text {ro }}\right)$ is defined as potential precipitation minus potential recharge. Palmer [23] assigned potential precipitation as being equal to AWC [47]. Hence,

$$
P_{r o}=\mathrm{AWC}-P_{r}=S_{s}+S_{u},
$$

where AWC is the available moisture capacity in soil layer, $P_{\mathrm{ls}}$ and $P_{\mathrm{lu}}$ are losses on surface and underlying soil layers, and $S_{s}$ and $S_{u}$ are the amount of available moisture stored in soil surface and underlying layers at the beginning of the month. Coefficients $\alpha, \beta, \gamma$, and $\delta$ can be computed using following equations [47]:

$$
\begin{gathered}
\alpha_{j}=\frac{\overline{\mathrm{ET}_{j}}}{\overline{P_{\mathrm{et}_{j}}}}, \\
\beta_{j}=\frac{\overline{R_{j}}}{\overline{P_{\mathrm{r}_{j}}}} \\
\gamma_{j}=\frac{\overline{\mathrm{RO}_{j}}}{\overline{\mathrm{Po}_{\mathrm{j}}}}, \\
\delta_{j}=\frac{\overline{L_{j}}}{\overline{P_{\mathrm{l}_{j}}}}
\end{gathered}
$$

where, $j=1,2, \ldots .12$ and $\overline{\mathrm{ET}_{j}}, \overline{R_{j}}, \overline{\mathrm{RO}_{j}}, \overline{L_{j}} \overline{P_{\text {et }}}, \overline{P_{\mathrm{r}_{j}}}, \overline{P_{\text {ro }}}$ and $\overline{P_{1 j}}$ are average values of month ' $j$ ' for evapotranspiration, recharge, runoff and loss and potential evapotranspiration, potential recharge, potential runoff, and potential loss, respectively. Monthly moisture anomaly is the disparity between measured precipitation and $P_{c}$. It is then multiplied by standardising factor at different sizes (can be designed to account for the variations in climate, using climatic parameters, such as long-term mean precipitation and temperature in this case), and the product is called the moisture anomaly index $Z$. For a month $t, Z$ can be computed as follows:

$$
Z(t)=K_{t} \times\left(P-P_{c}\right),
$$

where $P$ is the precipitation of month and $K_{t}$ is a weighing factor. For a detailed computation procedure $K_{t}$, readers are suggested to refer to Alley [47]. Palmer [23] suggested that a more appropriate moisture anomaly index $(X)$ can be given in the following form [47]:

$$
X(t)=c X(t-1)+\frac{Z(t)}{3},
$$

where first month, $X(t-1)=0$. Palmer [23] determined the values of $c=0.897$. Thus, the final expression will be

$$
X(t)=0.897 X(t-1)+0.33 Z(t),
$$


TABLE 2: IMD classification of drought.

\begin{tabular}{lcc}
\hline Intensity of meteorological drought & \% of departure from normal rainfall & Code \\
\hline No drought & 0.0 or above & M0 \\
Mild drought & 0.0 to -25.0 & M1 \\
Moderate drought & -25.0 to -50.0 & M2 \\
Severe drought & -50.0 or above & M3 \\
\hline
\end{tabular}

Source: [44].

where $X(i)$ is the PDI of the $t^{\text {th }}$ month. As per the coefficient values, the current month's PDI is just one-third of the current month's precipitation deficit and almost a tenth of the previous month's PDI. In other words, a month's PDI acts as a long-term memory of moisture content from the previous month. The PDI measurement requires both precipitation and temperature data since evapotranspiration estimation is involved.

The PDI has several advantages compared with other drought indices. It considers soil moisture content at a monthly time interval along with meteorological data. It allows also spatial and temporal representation of historical droughts. There is some limitation also such that it (a) simplifies soil representation, (b) estimates possible evapotranspiration using the simplified Thornthwaite process [46], and (c) ignores precipitation that falls as snow and snow cover [48].

PDI values can be categorised into eleven groups, as shown in Table 3. The PDI values can be categorised into eleven categories: highly wet (EW), quite wet (VW), mildly wet (SW), incipient wet spell (IWS), near normal (NN), incipient dry spell (IDS), mild drought (MiD), moderate dry (MoD), severely dry (SD), and exceptionally dry (ED).

2.4. Standardized Precipitation Index. Standardized precipitation index (SPI) [25] is a measure of meteorological drought. It is computed from the available rainfall data for a given duration. For transforming precipitation values to SPI values, following steps have been adopted.

(a) The mean of the precipitation values is calculated and adjusted to zero.

(b) The precipitation standard deviation is adjusted to 1.0 .

(c) Skewness of the existing data is readjusted to zero.

(d) SPI values now may be interpreted with mean as 0 with standard deviation 1.0 .

(e) Mean of precipitations can be computed as follows:

$$
\operatorname{Mean}\left(\overline{X_{\mathrm{im}}}\right)=\frac{\sum\left(X_{i j}\right)}{N},
$$

where $N$ is the number of precipitation observations, $X_{i j}$ is the seasonal precipitation at the $i^{\text {th }}$ rain gauge station and the $j^{\text {th }}$ observation, $\bar{X}_{\text {im }}$ is long-term seasonal mean, and $s$ is the standard deviation for precipitation dataset, computed as follows:

$$
s=\sqrt{\frac{\left(X_{i j}-\bar{X}_{\mathrm{im}}\right)}{N}} .
$$

(f) The skewness of the given dataset is computed as follows:

$$
\text { Skew }=\frac{1}{(N-1)(N-2)} \sum \frac{\left(X_{i j}-\bar{X}_{\mathrm{im}}\right)^{3}}{s} \text {. }
$$

(g) The precipitation values are converted to lognormal values in terms of statistical value $U$ as follows:

$$
\begin{aligned}
\text { Long term mean } & =\ln \left(\bar{X}_{\mathrm{im}}\right), \\
U & =\bar{X}_{\mathrm{im}}-\frac{\sum \ln \left(\bar{X}_{\mathrm{im}}\right)}{N} .
\end{aligned}
$$

(h) Shape parameter is calculated as follows:

$$
\text { Shape parameter }=\beta=\frac{1+\sqrt{1+(4 U / 3)}}{4 U} .
$$

(i) Scale parameter is calculated as follows:

$$
\text { Scale parameter }=\alpha=\frac{\bar{X}_{\mathrm{im}}}{\beta} \text {. }
$$

(j) Equations (15a)-(15d) and (15f) are solved using Excel's built-in functions. The resulting parameters are then used to measure the accumulated likelihood of a precipitation occurrence that has been observed. The cumulative probability is given by

$$
G(x)=\frac{\int_{0}^{x} x^{\alpha-1} e^{-(x / \beta)}}{\beta^{\alpha} \Gamma(\alpha)} .
$$

Since the gamma function is undefined for $x=0$ and because a precipitation distribution may contain zeros, the cumulative likelihood can be calculated as

$$
H(x)=q+(1-q) G(x) .
$$

(k) The SPI values are determined by converting the accumulated likelihood $H(x)$ to standard normal random variable $Z$ (mean $=0$, standard deviation values.

(l) The approximate conversion [48] is adopted as

$$
Z=\mathrm{SPI}=-\left[t-\frac{C_{0}+C_{1} t+C_{2} t^{2}}{1+d_{1} t+d_{2} t^{2}+d_{3} t^{3}}\right],
$$


TABLe 3: Drought classification based on PDI values.

\begin{tabular}{|c|c|c|c|c|c|c|c|c|c|c|c|}
\hline & $\begin{array}{c}\text { Extremely } \\
\text { wet } \\
(\mathrm{EW})\end{array}$ & $\begin{array}{l}\text { Very } \\
\text { wet }\end{array}$ & $\begin{array}{c}\text { Moderately } \\
\text { wet } \\
\text { (MW }\end{array}$ & $\begin{array}{c}\text { Slightly } \\
\text { wet } \\
(\mathrm{SW})\end{array}$ & $\begin{array}{c}\text { Incipient } \\
\text { wet spell } \\
\text { (IWS) }\end{array}$ & $\begin{array}{c}\text { Near } \\
\text { normal } \\
(\mathrm{NN})\end{array}$ & $\begin{array}{c}\text { Incipient } \\
\text { dry spell } \\
\text { (IDS) }\end{array}$ & $\begin{array}{c}\text { Mild } \\
\text { drought } \\
(\mathrm{MiD})\end{array}$ & $\begin{array}{l}\text { ate } \\
\text { ht } \\
\text { ) }\end{array}$ & $\begin{array}{r}\mathrm{Se} \\
\mathrm{drc} \\
(\end{array}$ & $\begin{array}{l}\text { me } \\
\text { ght } \\
\text { ) }\end{array}$ \\
\hline & & 3.99 & & $\begin{array}{c}1.0 \text { to } \\
1.99\end{array}$ & & & & & & & $\begin{array}{c}-4.0 \text { or } \\
\text { less }\end{array}$ \\
\hline
\end{tabular}

Source: $[7,53,55,59]$.

for $0<H(x) \leq 0.50$ and

$$
Z=\mathrm{SPI}=+\left[t-\frac{C_{0}+C_{1} t+C_{2} t^{2}}{1+d_{1} t+d_{2} t^{2}+d_{3} t^{3}}\right],
$$

for $0.5<H(x) \leq 1.00$,

where

$t=\sqrt{\ln \left(\frac{1}{H(x)^{2}}\right)}, \quad$ for $0<H(x)$,

$t=\sqrt{\ln \left(\frac{1}{1-H(x)^{2}}\right)}, \quad$ for $0.5<H(x) \leq 1$,

where $C_{0}=2.515517, C_{1}=0.802583, C_{2}=0.010328$, $D_{1}=1.432788, D_{2}=0.189269$, and $D_{3}=0.001308$.

The SPI values may be classified into seven categories, namely, extremely wet, very wet, moderately wet, near normal, moderately dry, severely dry, and extremely dry as shown in Table 4.

2.5. Identification of Drought Events Using Log Pearson Type III Distribution Technique. A log Pearson type III distribution was assumed to fit nicely [49] to achieve a more meaningful interpretation because the meteorological drought indicator values include precipitation data. The obvious approach to apply Pearson III is to use the sample's mean, standard deviation, and skewness as statistical parameters. Sorting the sample and assigning rough likelihood estimates to each point based on its rank is a routine procedure [50]. An empirical approach with probability estimates uses skewness functions as per equations provided in (20a)-(20c). The skewness functions [50] in the Microsoft Excel spreadsheet return estimates of $\gamma_{1}$ is as follows:

$$
\begin{aligned}
& P I I I(x)=\Gamma D(x-\Upsilon, \alpha, \beta) ; \gamma_{1}>0, \\
& P I I I(x)=\Gamma D(\Upsilon-x, \alpha, \beta) ; \gamma_{1}<0 .
\end{aligned}
$$

One can combine the previously mentioned two expressions as follows:

$$
\operatorname{PIII}(x)=\Gamma D\left((x-\Upsilon) \cdot \frac{\gamma_{1}}{\left|\gamma_{1}\right|}, \alpha, \beta\right),
$$

where $\Gamma D$ is the standard gamma distribution function and 'shape' $\alpha=4 / \gamma_{1}^{2}$; "scale" $\beta=\left|s \gamma_{1} / 2\right|(\beta=s / \sqrt{\alpha})$; "shift" $\gamma=$ $\mu-2 s / \gamma_{1}\left(\gamma=\mu \pm \alpha \beta\right.$, depending upon the sign of $\gamma_{1}$, where $\mu, s$, and $\gamma_{1}$ are the mean, standard deviation, and skewness of the distribution.
The logarithmic transformation is written as follows:

$$
P \operatorname{III}(x)=\Gamma D\left(\log (x-\Upsilon) \cdot \frac{\gamma_{1}}{\left|\gamma_{1}\right|}, \alpha, \beta\right),
$$

where $\alpha, \beta, \Upsilon$ and $\mu, s, \gamma_{1}$ are defined in terms of $\log (x)$ instead of $x$ (base is 10). If $\gamma_{1}$ is exactly 0 , the distribution is not specified for $x$ outside the transition, which is the lower bound for positive skew and the upper bound for negative skew. There are built-in functions in Excel software.

\section{Study Area and Data Used}

Tamil Nadu in South Eastern region of India is the eleventh largest state of India with having geographical area of $130058 \mathrm{~km}^{2} \quad$ (http://www.walkthroughindia.com/offbeat/ top-15-largest-states-india-geographical-area/) extending its length and breadth approximately between $8^{\circ} \mathrm{N}-14^{\circ} \mathrm{N}$, $76^{\circ} \mathrm{E}-81^{\circ} \mathrm{E}$. Tamil Nadu is having the third largest costal line of $940 \mathrm{~km}$ after Gujarat and Andhra Pradesh (https://en. wikipedia.org/wiki/Coastline_of_Tamil_Nadu). It is located on the inward side of the Western Ghats, resulting in relatively low rainfall compared with outward coastline of Western Ghats of Kerala state. Hence, drought like situation is prevalent on the part of Western Ghats, which lies in Western Tamil Nadu, whereas Karnataka is a landlocked state in India's southernmost region. It has a longitudinal extent of 74 degrees east to 78 degrees east and a latitudinal extent of 11 degrees north to 18 degrees north. It covers an area of $191791 \mathrm{~km}^{2}$ and is India's seventh largest state (http:// www.walkthroughindia.com/offbeat/top-15-largest-statesindia-geographical-area/).

The southernmost point of India (Indira Point) in Kanyakumari is also located in Tamil Nadu. Figure 1 representing the mean annual rainfall and temperature suggests that Karnataka has a relatively higher annual rainfall compared with Tamil Nadu with respect to districtwise distribution (Figure 2), but temperature patterns for those districts are distinct. The location map of study region containing both states is represented in Figure 2. Monthly precipitation and temperature data for all districts in Tamil Nadu and Karnataka were collected from India Water Portal (http://www.indiawaterportal.org/metdata/) for a span of 110 years (1901-2010).

Before utilising these data to further statistical analysis, such as Mann-Kendall test, a prewhitening technique is performed.

A detailed flowchart of stepwise procedure for the present study for rainfall characterisation is presented in Figure 3. 
TABLE 4: Drought classification based on SPI values.

\begin{tabular}{lccccccc}
\hline Classification Extremely wet & Very wet (VW) & $\begin{array}{c}\text { Moderately wet } \\
\text { (MW) }\end{array}$ & $\begin{array}{c}\text { Near normal } \\
\text { (NN) }\end{array}$ & $\begin{array}{c}\text { Moderately dry } \\
\text { (MD) }\end{array}$ & $\begin{array}{c}\text { Severely dry } \\
\text { (SD) }\end{array}$ & Extremely dry (ED) \\
\hline SPI value & $\geq 2$ & 1.5 to 1.99 & 1 to 1.49 & -0.99 to 0.99 & -1.0 to -1.49 & -1.5 to -1.99 & $\leq-2$
\end{tabular}

Source: $[55,59]$.

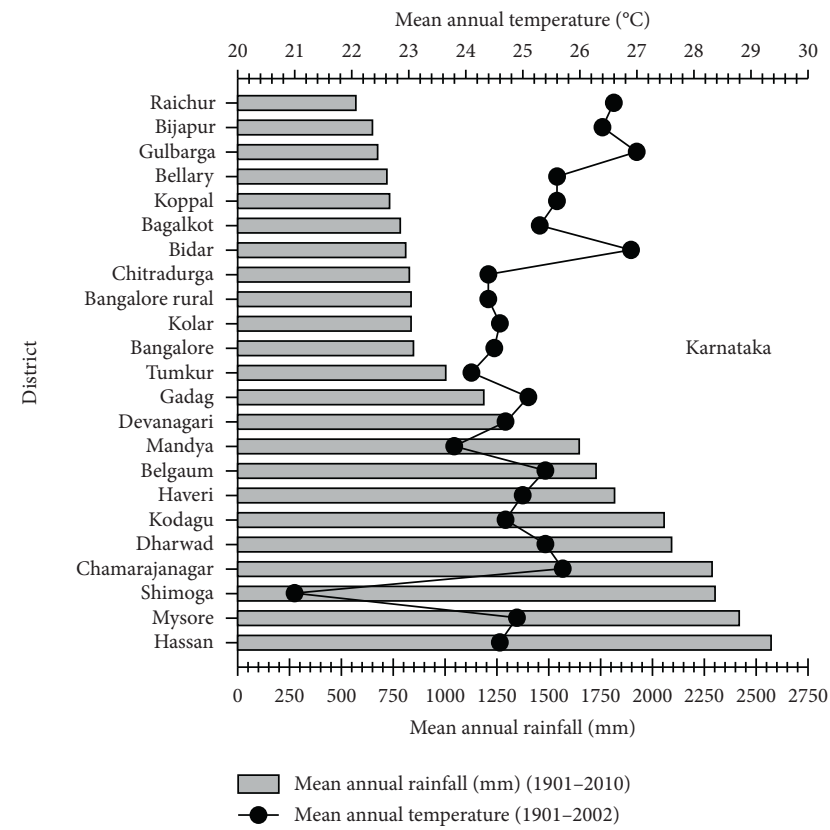

(a)

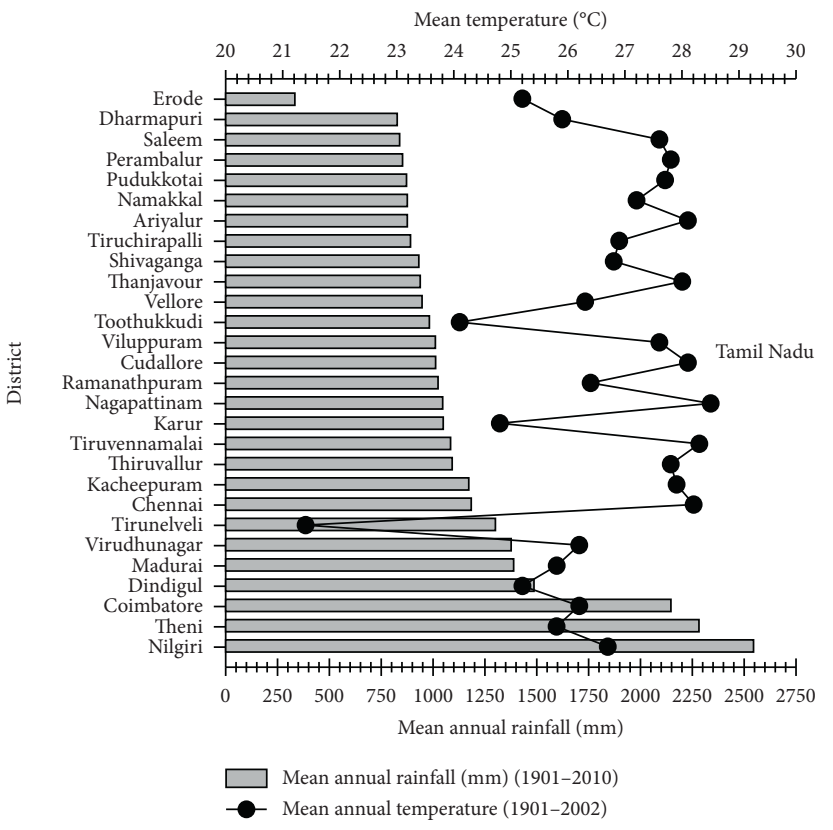

(b)

Figure 1: District-wise mean annual rainfall and mean annual temperature for two states.

\section{Results and Analysis}

\subsection{Rainfall Trend Analysis}

4.1.1. Annual Rainfall Classification. Data analysis were worked out in steps to get better insight into drought conditions considering rainfall data for 110 year for both regions. Monthly rainfall gauge data for each district or climatic division of both regions were acquired, and annual rainfall was computed for 110 years. Rainfall datasets for 110 years for Tamil Nadu and Karnataka were segregated according to regional rainfall classification [51] and presented in Table 5 to understand the relative wetness status of two study regions. Number of years within study time of 110 years for annual rainfall under low, medium, high, and very high ranges for both states were counted, statistically analysed, and presented in a graphical form in Figure 4 and corresponding distribution shown in map represented in Figure 5.

\subsubsection{Rainfall Trend Analysis Using Mann-Kendall Test.} Rainfall data of all district gauge stations for two states were further analysed using Mann-Kendall test and trend of rainfall variation using linear regression techniques had been worked out. District stations, which registered highest decreasing trends, were identified and corresponding trends with data points were graphically represented in Figures 6 and 7 along with monthly rainfall data points for a study span of 110 years for Karnataka and Tamil Nadu, respectively. The district gauge stations with decreasing trends were segregated after performing Mann-Kendall test and represented in a tabular form in Table 6, with all its indicators and parameters. The null hypothesis $H_{0}$, which denotes no trend, cannot be rejected if the computed $p$ value is greater than the significance value alpha $=0.05$, and vice versa. The correction for continuity has been applied. Sen's slope test was also conducted, and Sen's slopes, which indicate true slope of data points, are also presented in Table 6.

Figure 8 depicts a map of Karnataka and Tamil Nadu that shows all the districts with declining and rising rainfall trends throughout the study time (1901-2010). It is noticeable that a slight decrease or insignificant increase in rainfall in most districts of Tamil Nadu would have a larger impact on drought vulnerability with respect to that of Karnataka, and the mean annual rainfall in Tamil Nadu region is comparatively lower than that of Karnataka region. It is pertinent to mention that, for the period of study (1901-2010) based on annual rainfall classification, there were either high rainfalls in 38 years or medium rainfalls in 15 years. However, there were low rainfalls only for 15 years in some of the districts of Karnataka. Very high rainfalls were recorded in 42 years for some districts of Karnataka.

According to the Annual Rainfall Classification, four districts have low rainfall (high risk of drought), seven 


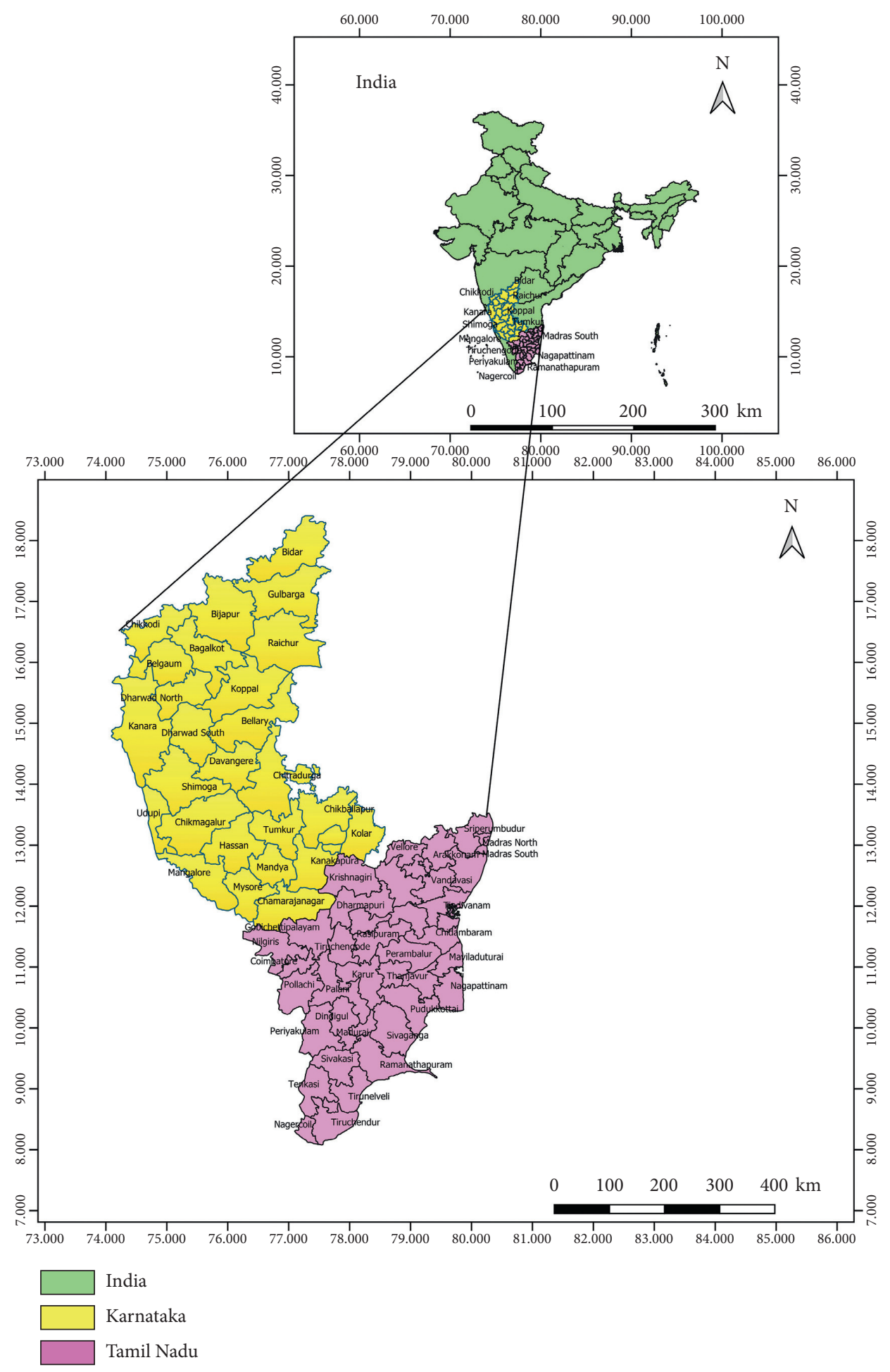

Figure 2: Location map representing Karnataka and Tamil Nadu states of India.

districts have medium rainfall (moderate risk of drought), five districts have moderate rainfall (low risk of drought), and six districts have very high rainfall (very low risk of drought). In Tamil Nadu region, for the period of study (1901-2010) in general, there were either high rainfalls in 35 years or medium rainfalls in 45 years. However, there have been poor rainfalls in some Tamil Nadu districts for the past
16 years. The districts of Tamil Nadu have seen the highest rainfall in 14 years. According to the Annual Rainfall Classification, one district has poor rainfall (extremely high chance of drought), 18 districts have medium rainfall (high risk of drought), six districts have high rainfall (low risk of drought), and only three districts have very high rainfall (very low risk of drought). While rainfall data analysis using 


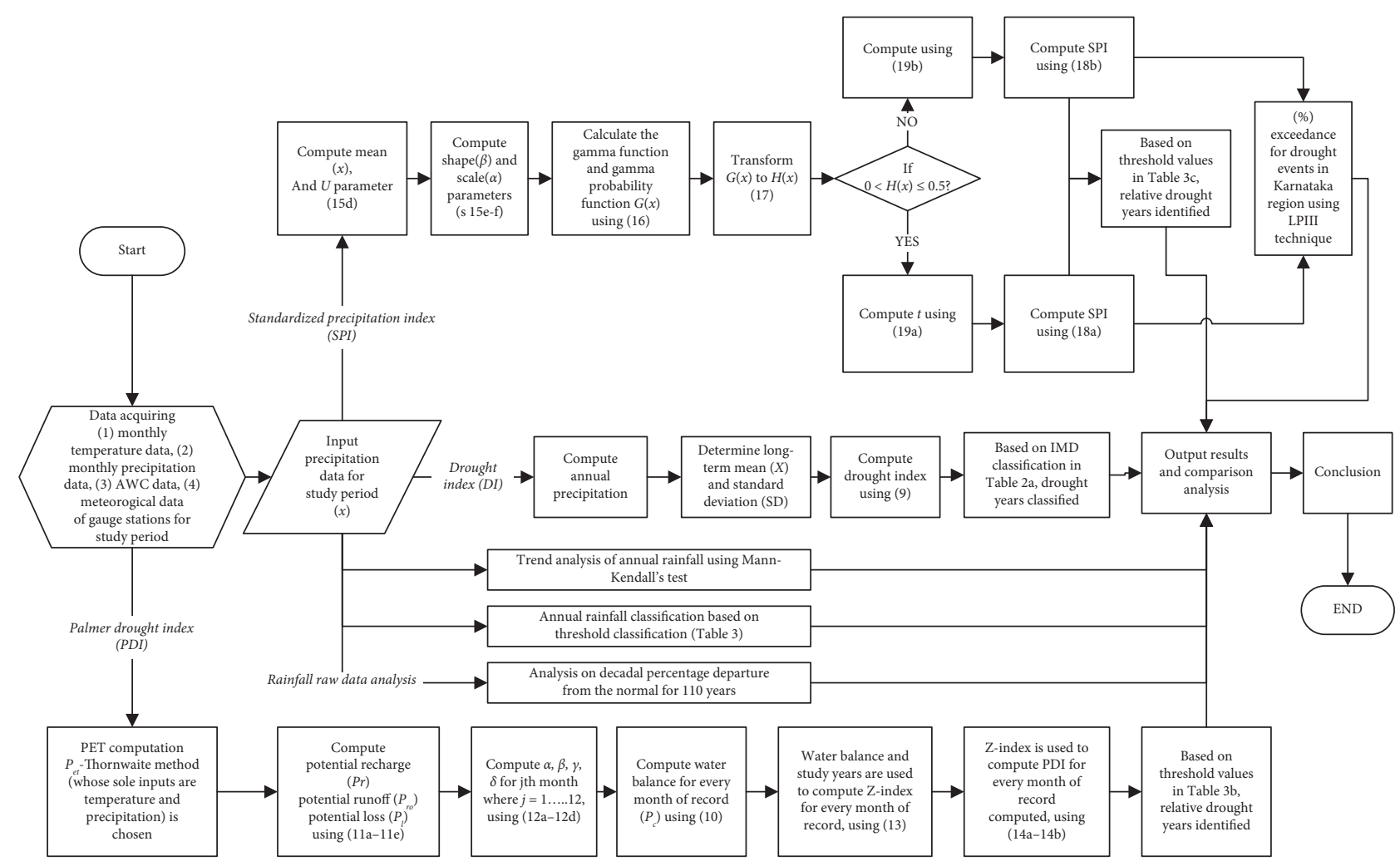

FIGURE 3: Flowchart showing stepwise procedure for the present study.

TABLE 5: Ranges of rainfall distribution.

\begin{tabular}{lc}
\hline Classification & Range (in $\mathrm{mm}$ ) \\
\hline Low rainfall & $<750$ \\
Medium rainfall & 750 to 1125 \\
High rainfall & 1125 to 2000 \\
Very high rainfall & $>2000$ \\
\hline
\end{tabular}

Source: [51].

annual rainfall classification and trend analysis may provide a qualitative indicator of meteorological processes, adequate meteorological drought characterisation necessitates more in-depth analysis.

4.1.3. Decadal Percentage Departure from the Normal. It is well established that the summer monsoon rainfall in India exhibits multidecadal variability with a clustering of rainy or dry irregularities [52]. To separate low frequency behaviour, 10 -year running means of annual rainfall were computed to analyse epochs of above and below normal rainfall. These epochs of above and below normal rainfall are depicted in Figures 9 and 10 for six meteorological district stations in Karnataka and Tamil Nadu, respectively, with declining rainfall trends.

\subsection{Drought Analysis}

4.2.1. Drought Index Analysis. Based on Annual Rainfall Classification (Figure 4), there were 11-12 districts in Karnataka with high/very rainfall (low chance of drought) for many of the years, while nearly 19 districts in Tamil Nadu had medium or low rainfall for most of the years (high risk of drought) during the 110-year study period (1901-2010). However, from this analysis, it is possible to extract a reasonably qualitative concept. If only the classification is considered, the districts of Dharwad, Mysore, Belgaum, Devanagari, and Bagalkot represent a significant number of years with heavy rainfall. Nilgiri and Vidyut Nagar in Tamil $\mathrm{Nadu}$, likewise, received a huge amount of rain. However, according to the statistical trend analysis described in the previous section, rainfall in these districts was on a decreasing trend, resulting in meteorological drought, which supported the realistic scenario for those districts. As a result, it is obvious that the annual rainfall classification has drawbacks and is no longer capable of forecasting a concrete drought condition in the study region. To get a better understanding of the drought condition, a drought indicator may be used that includes the average effects of rainfall decrease or rise over a longer period.

In context of this, the drought index (IMD 1971) was estimated using (9) using monthly rainfall data spanning 110 years [49]. According to the threshold values given in Table 2, 14 districts in Karnataka had an average of 44 mild drought event years, 10 moderate drought event years, and two extreme drought event years over the course of 110 years. Similarly, in Tamil Nadu, an average of 46 times of mild drought, six times of moderate drought, and one time of severe drought in 28 districts were found during the study period (1901 to 2010). The only difference that can be readily inferred is that \% areal extend of drought condition was 


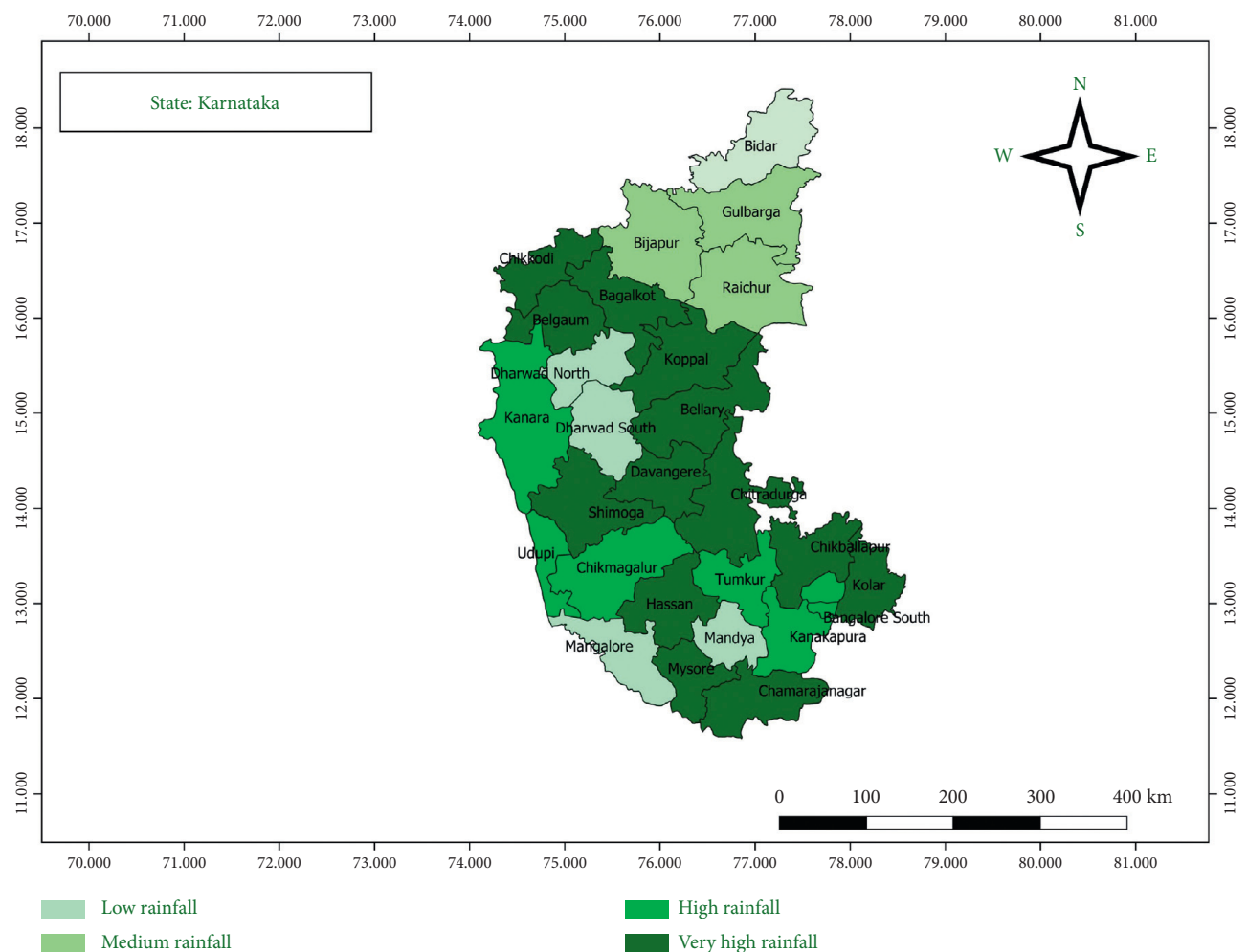

(a)

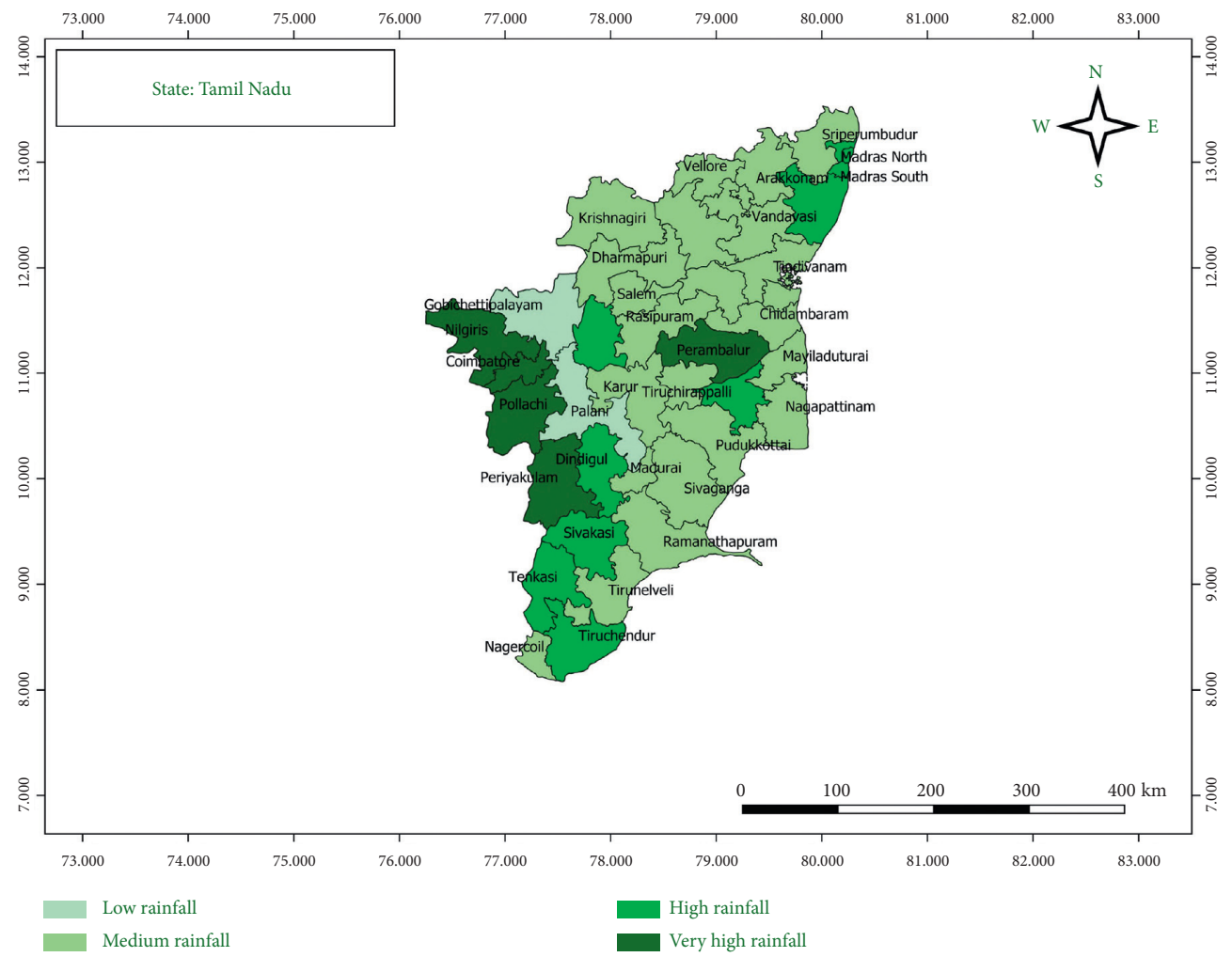

(b)

FIGURE 4: Rainfall distribution of study area based on annual rainfall classification. 


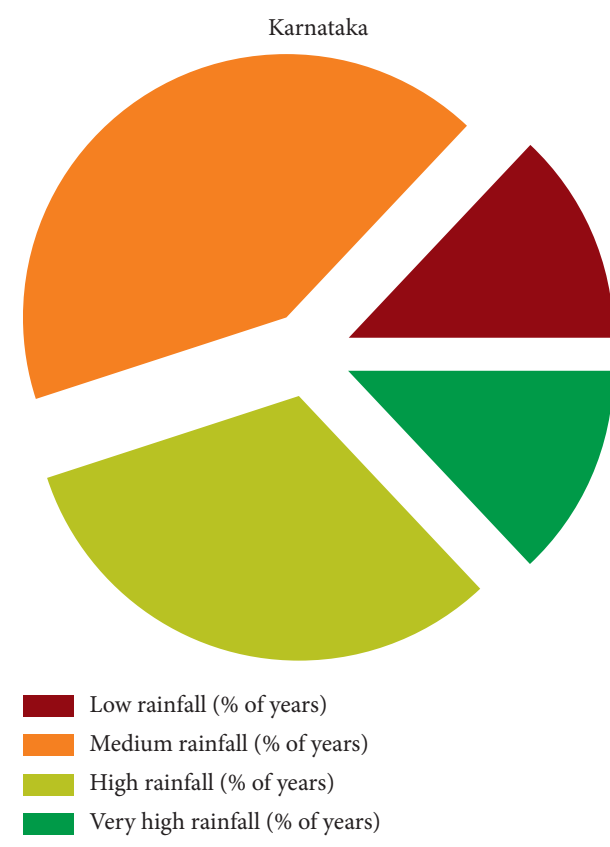

(a)

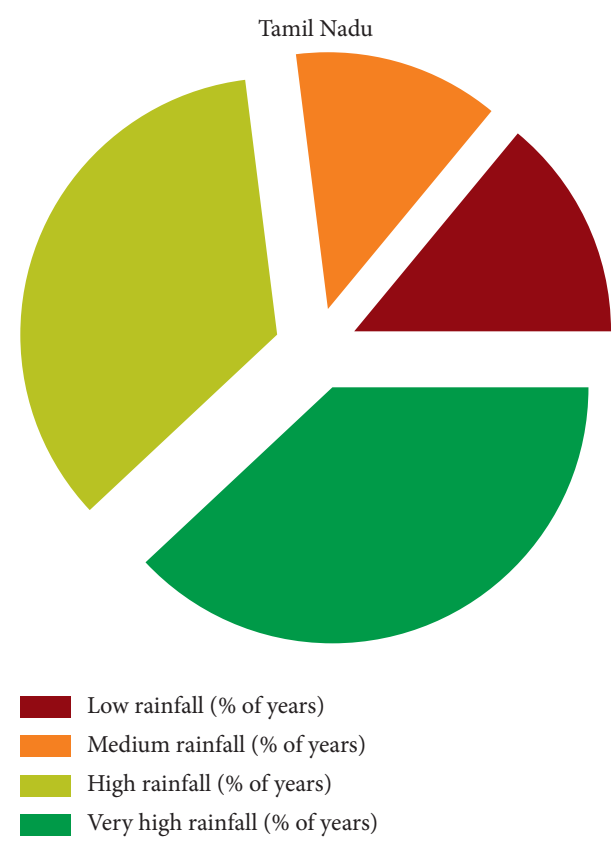

(b)

Figure 5: \% number of year verses rainfall classification for all districts.

more in Tamil Nadu than that of Karnataka due to low to medium annual rainfall recorded in Tamil Nadu. The areal extend of drought affected of Karnataka and Tamil Nadu as per DI values is shown in Figure 11 [49].

4.2.2. Palmer Drought Index Analysis. Palmer index values were calculated using climatic data for precipitation and temperature [23] and methods described in Section 2.3. The practical calculation illustrates the complexity of the PDI [45]. The simplified Thornthwaite method [46] was used for estimating potential evapotranspiration. Thus, PDI is calculated from precipitation deficits for monitoring long-term drought conditions with 12-month intervals to compare it with twelve-month SPI values. Based on computed PDI values, event years with various intensities of meteorological drought, wet, and near normal (NN) conditions based on threshold values presented in Table 3.

4.2.3. Standardized Precipitation Index Analysis. For available precipitation data, the standardized precipitation index (SPI) $[25,53]$, which integrates seasonal variation in rainfall and can be statistically analysed by fitting to the Gamma distribution function [54], was computed using the methods provided in Section 2.4. SPI values for the 12month period were based primarily on their suitability for water resource studies [54-56]. The number of event years was found to be under drought situation in categories, such as moderately dry, severely dry, and extremely dry, for all meteorological district stations of both states. It is presented in Table 7 with statistical mean and standard deviations and is graphically represented in Figure 12.
4.2.4. Comparison of Three Drought Indices for Two Study Regions. District-wise computed drought indicators, namely DI, PDI, and SPI, for both states for the study period were compared [49] with a percentage mean frequency of occurrence with their indicator-wise threshold values and presented in Figure 13. Near normal (NN) condition was prevailing more than 50 percent of the study period for all considered indices. However, there were visible differences of estimated frequencies of occurrence for mild-to-severe drought conditions for each index analysis of two study regions. Noticeably, the pattern is coherent in case of DI, PDI, and SPI with small variations. Furthermore, when comparing severe drought conditions, both indicators show that Karnataka is more vulnerable than Tamil Nadu. Corresponding standard deviation values are also plotted to have insight into the spread of the variation of severity for all districts of each state (Figure 13).

The computed values for yearly DI, PDI, and SPI for two chosen districts with recent yearly rainfall deficit for Karnataka and Tamil Nadu are presented here as Figures 14 and 15 for illustration.

4.2.5. Identification of Drought Events Using Log Pearson Type III Distribution Technique for Karnataka. The computed SPI data for Karnataka were then analysed further to assess the probability of exceeding SPI threshold values in terms of severity. For 110 years (1901-2010), the log Pearson Type III distribution on the adjusted nonzero drought deficit in terms of SPI, namely, scaled SPI, using a nonzero drought deficit parameter (SPI threshold value plus five as shown in Table 8) was computed. Furthermore, the percentage probability of exceedance for drought deficit 


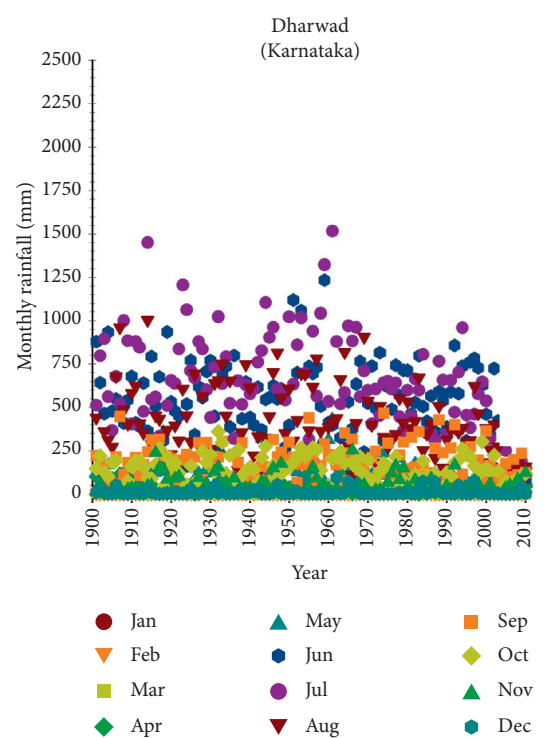

(a)

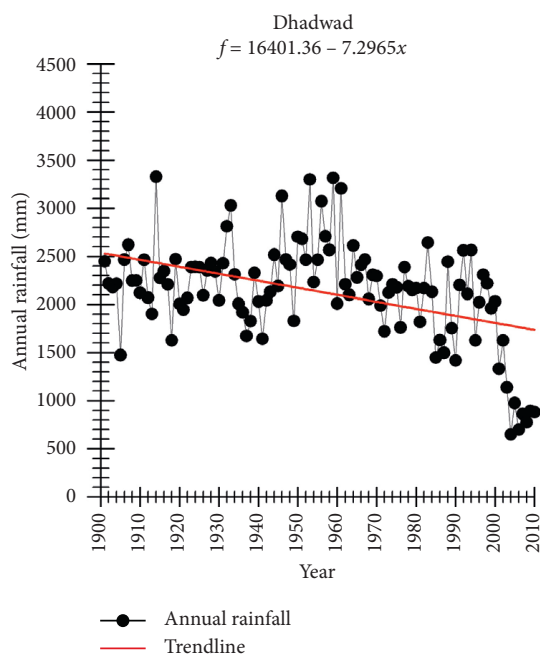

(d)

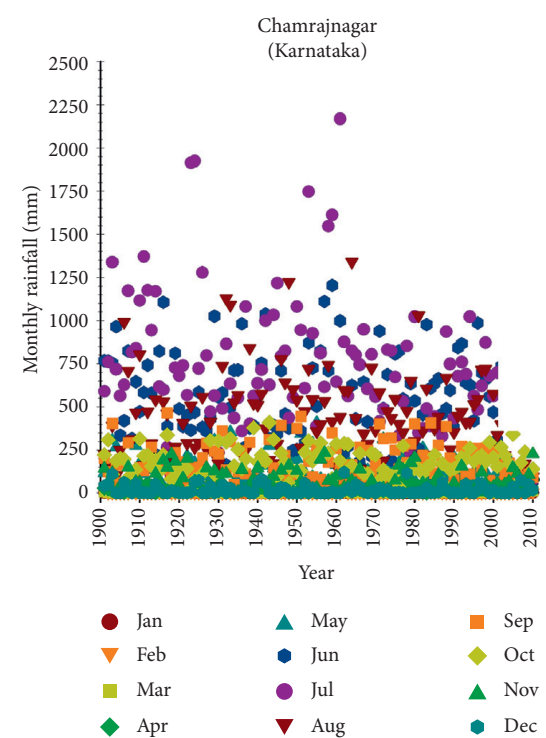

(b)

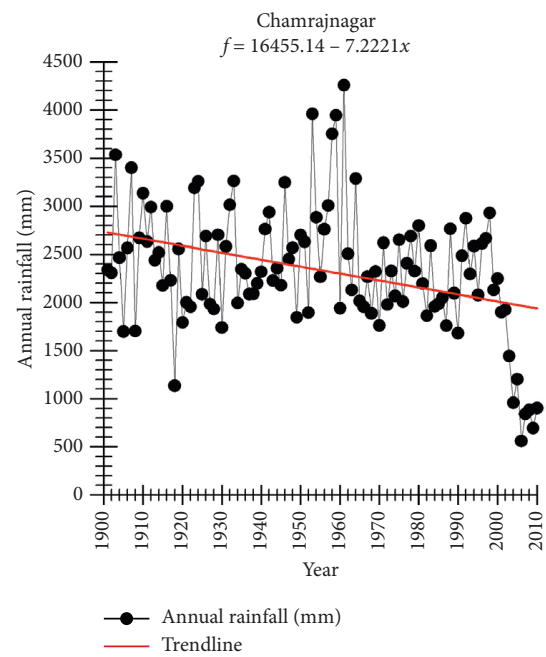

(e)

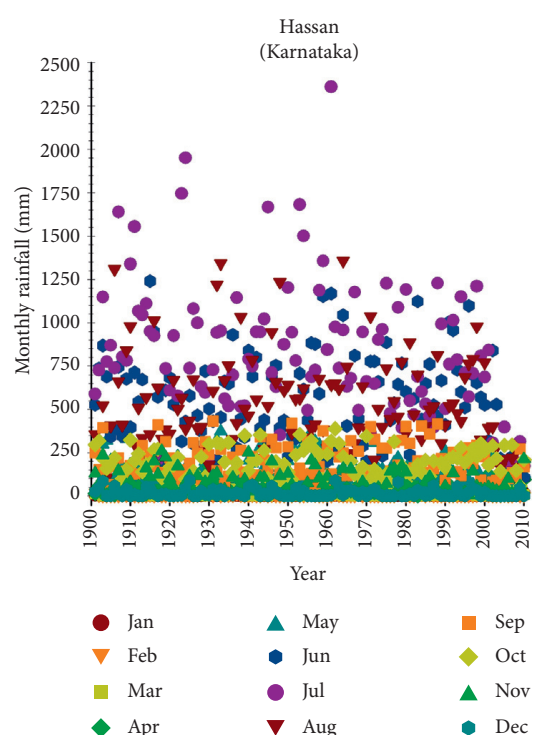

(c)

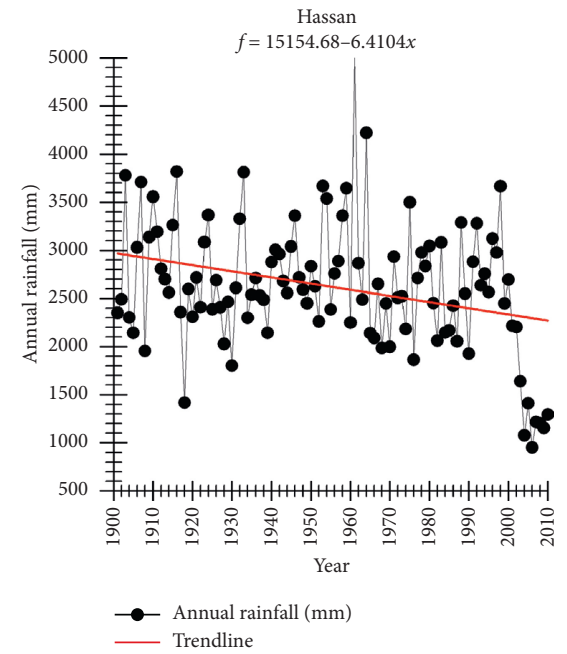

(f)

FIgURE 6: District with higher decreasing rainfall trends in Karnataka.

in all 23 Karnataka districts was determined using skewness functions as per the equations provided in (20a)-(20c).

As a result, for the 110 years (1901-2010), the modified nonzero drought deficit in terms of SPI, namely, scaled SPI, using a nonzero drought deficit parameter (SPI threshold value plus five as shown in Table 8) was computed using the log Pearson Type III distribution.

For moderate, severe, and extreme drought events, the derived data set for all 23 districts of the Karnataka region in the Indian Peninsula is graphically represented in Figure 16 based on the threshold values (presented in Table 8).

Vulnerability to drought, in general, varies spatially and is broadly assessed by natural factors, such as intensity and magnitude of rainfall scarcity and drought hazards, which lead to its susceptibility, and by social factors, which lead to exposure, coping capacity, and adaptive capability.
Accurate quantification of vulnerability is quite complex; however, relative assessment of vulnerability may be worked out based on estimation of drought indicators, such as SPI or DI.

From the computed exceedance probabilities for moderate, severe, and extreme drought ranges (shown in Figure 12), corresponding return period ( $T$ in years) were computed (return period $=1$ /exceedance probability). Furthermore, for each drought severity class, return period intervals were fixed based on the range between maximum and minimum return period values. For example, return period intervals were fixed as [3-20, 57] [22-44), (44-51] (with corresponding vulnerability assigned values 4, 3, 2, and 1 in decreasing vulnerability on four-point scale) for a range between 3 and 51 years for moderate drought (MD). Similarly, for severe drought (SD) and extreme drought (ED) with corresponding return period ranges (5-2500) and 


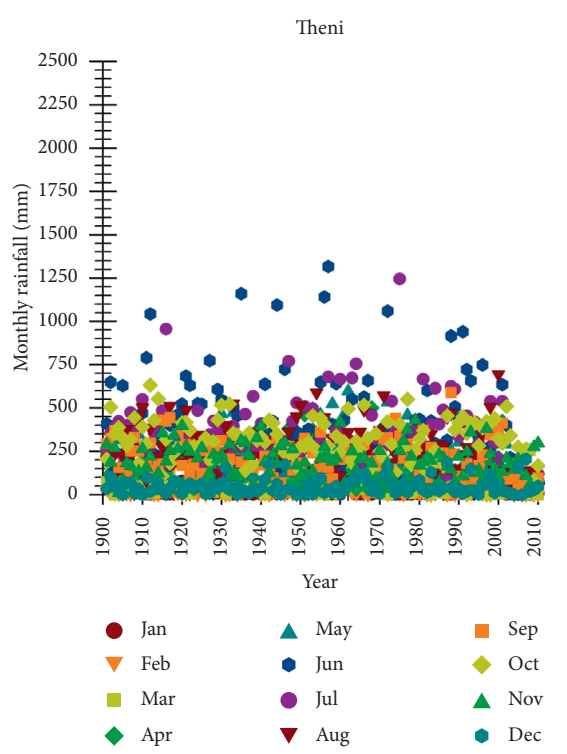

(a)

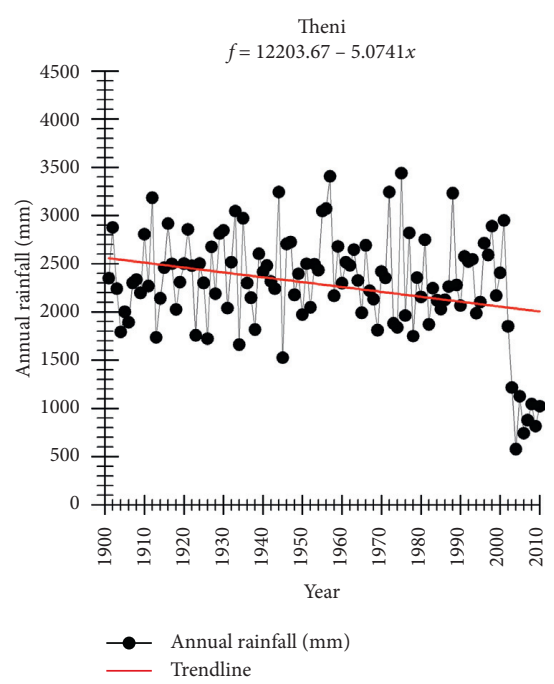

(d)

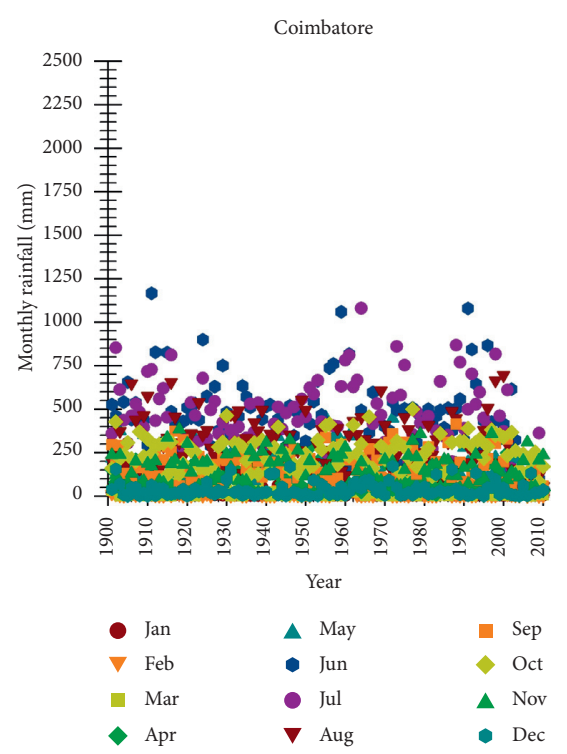

(b)

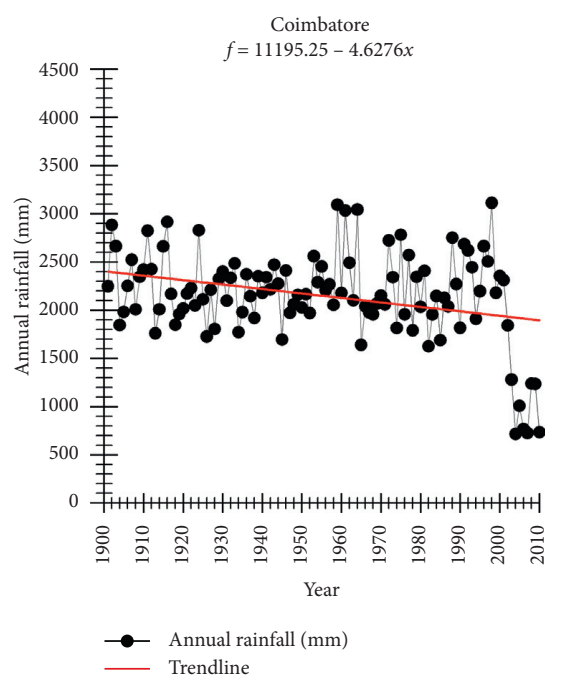

(e)

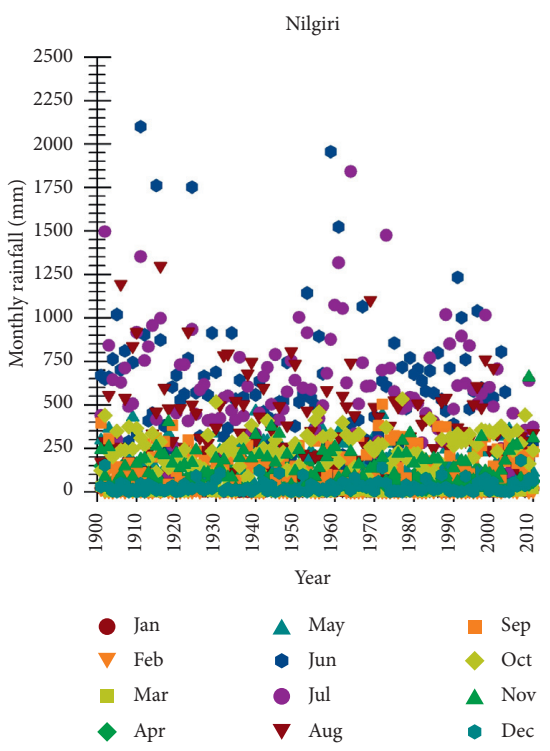

(c)

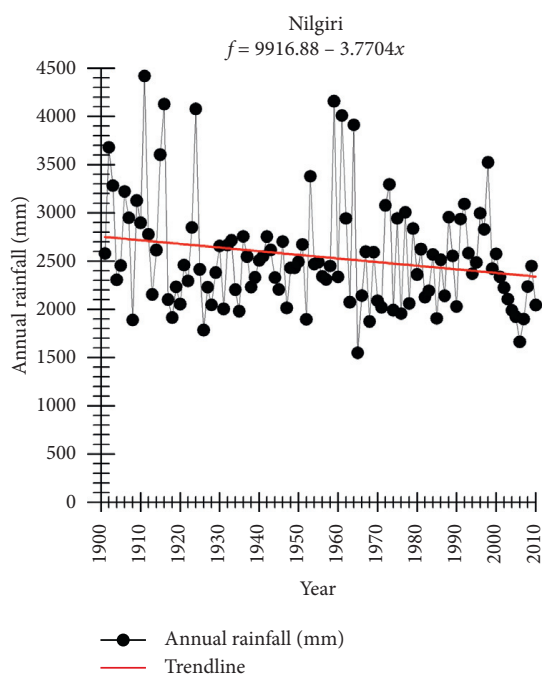

(f)

Figure 7: Districts with higher decreasing rainfall trends in Tamil Nadu.

(65-15000), respectively, return period intervals were chosen as [5-250], [251-800], [900-1600], [1700-2500] (with corresponding vulnerability assigned values $4,3,2$ and 1 in decreasing vulnerability on four-point scale) and [65-1000], [1100-2000], [2100-9000], [10000-15000] (with corresponding vulnerability assigned values 4, 3, 2 and 1 in decreasing vulnerability on four-point scale). For each drought class (moderate, severe, and extreme), each district had a unique return period computed from corresponding exceedance probability (Figure 16), and vulnerability assigned values ranging from 4 to 1 are worked out depending on the interval within which a return period is lying for that district. Thus, for each district relative vulnerability the threshold ranges on drought were determined and mapped using GIS tools as shown in Figures 17-19.

\section{Discussion}

\subsection{Rainfall Trend}

5.1.1. Annual Rainfall Classification. It is readily inferred from Figures 4 and 5 that relative wetness in Karnataka region was higher compared with Tamil Nadu with respect to areal extend; however, recurrences suggest vice versa. In Karnataka region, three district stations were exhibiting high annual rainfall status, whereas Tamil Nadu had a majority of district having medium annual rainfall for the last 110 years of time span. This is due to the fact that Tamil Nadu lies in Eastern zone of Western Ghats.

The Eastern Ghats lying in Tamil Nadu are irregular, broken, and smaller in heights compared to Western Ghats. However, moisture laden winds reach on the Eastern part leading to moderately good rainfall condition in this region. 
TABLE 6: Consolidated Mann-Kendall test results (of decreasing rainfall trends) for study regions for 110 years (significance level $\alpha=0.05$ ).

\begin{tabular}{|c|c|c|c|c|c|c|c|c|c|c|}
\hline $\begin{array}{l}\text { S. } \\
\text { No. }\end{array}$ & $\begin{array}{l}\text { District gauge } \\
\text { station }\end{array}$ & $\begin{array}{c}\text { Observation } \\
\text { without missing } \\
\text { data }\end{array}$ & $\underset{(\mathrm{mm})}{\operatorname{Minimum}}$ & $\begin{array}{l}\text { Maximum } \\
(\mathrm{mm})\end{array}$ & Mean & $\begin{array}{c}\text { Std. } \\
\text { deviation }\end{array}$ & $\begin{array}{c}\text { Kendall's } \\
\text { tau }\end{array}$ & $S$ & $\begin{array}{l}p \text { value } \\
\text { (two- } \\
\text { tailed) }\end{array}$ & $\begin{array}{l}\text { Sen's } \\
\text { slope }\end{array}$ \\
\hline \multicolumn{11}{|c|}{ Karnataka } \\
\hline 1 & Chamrajnagar & 110 & 558 & 4255 & 2332 & 660.24 & -0.203 & -1219 & 0.002 & -6.02 \\
\hline 2 & Dharwad & 110 & 648 & 3326 & 2133 & 533.25 & -0.257 & -1543 & $<0.0001$ & -5.39 \\
\hline 3 & Hassan & 110 & 948 & 5024 & 2619 & 676.21 & -0.171 & -1025 & 0.008 & -5.38 \\
\hline 4 & Mysore & 110 & 699 & 4531 & 2464 & 688.77 & -0.140 & -839 & 0.030 & -4.28 \\
\hline 5 & Haveri & 110 & 707 & 2741 & 1848 & 413.86 & -0.197 & -1181 & 0.002 & -4.00 \\
\hline 6 & Belgaum & 110 & 801 & 2853 & 1756 & 392.15 & -0.152 & -911 & 0.019 & -2.96 \\
\hline 7 & Devanagari & 110 & 571 & 1971 & 1331 & 294.33 & -0.191 & -1147 & 0.003 & -2.74 \\
\hline 8 & Shimoga & 110 & 1265 & 3838 & 2302 & 453.23 & -0.121 & -725 & 0.061 & -2.58 \\
\hline 9 & Gadag & 110 & 493 & 1805 & 1207 & 259.93 & -0.205 & -1231 & 0.001 & -2.48 \\
\hline 10 & Mandya & 110 & 486 & 2902 & 1676 & 426.00 & -0.100 & -601 & 0.121 & -1.95 \\
\hline 11 & Chitradurga & 110 & 499 & 1268 & 837 & 164.58 & -0.082 & -493 & 0.204 & -0.68 \\
\hline 12 & Tumkur & 110 & 584 & 1483 & 1005 & 188.71 & -0.0542 & -325 & 0.403 & -0.51 \\
\hline 13 & Bellary & 110 & 407 & 1086 & 725 & 137.97 & -0.032 & -191 & 0.624 & -0.18 \\
\hline \multicolumn{11}{|c|}{ Tamil Nadu } \\
\hline 1 & Theni & 110 & 576 & 3437 & 2281 & 554.69 & -0.1283 & -769 & 0.0473 & -3.32 \\
\hline 2 & The Nilgiris & 110 & 1545 & 4417 & 2544 & 566.64 & -0.1269 & -761 & 0.0496 & -3.03 \\
\hline 3 & Coimbatore & 110 & 714 & 3111 & 2146 & 471.88 & -0.1376 & -825 & 0.0333 & -2.82 \\
\hline 4 & Vidyutnagar & 110 & 536 & 2067 & 1376 & 299.05 & -0.1413 & -847 & 0.0289 & -2.09 \\
\hline 5 & Madurai & 110 & 743 & 2090 & 1389 & 296.22 & -0.1036 & -621 & 0.1093 & -1.56 \\
\hline 6 & Tirunelveli & 110 & 840 & 1834 & 1299 & 208.21 & -0.1179 & -707 & 0.0682 & -1.22 \\
\hline 7 & Dindigul & 110 & 800 & 2144 & 1487 & 260.13 & -0.0899 & -539 & 0.1646 & -1.17 \\
\hline 8 & Karur & 110 & 556 & 1513 & 1048 & 182.97 & -0.0565 & -339 & 0.3826 & -0.54 \\
\hline
\end{tabular}

Hence, one can even find large variation in rainfall intensities between Eastern and Western Tamil Nadu.

5.1.2. Rainfall Trend Using Mann-Kendall Test. Districts with decreasing trends are vividly depicted in map showing two states in Figure 8. From all the results shown, it can be inferred that number of districts with decreasing rainfall trend was 14, whereas, in Tamil Nadu districts with rainfall, decrease trend was 8. In Karnataka, the trend lines of districts with rainfall decrease were considerably higher with respect to that of Tamil Nadu. This behaviour is natural as the average annual rainfall in Karnataka was relatively higher side with respect to Tamil Nadu and may not necessarily indicate meteorological drought condition in Karnataka. However, insignificant or slight rainfall decrease in regions with low annual rainfall may come under meteorological drought condition as region is already water scarce in character.

From the obtained result, it may be inferred that Karnataka, being a rain-fed region, may have decreased rainfall trends in many districts; however, with respect to drought conditions, Tamil Nadu has more vulnerability with areal extends. It can be easily inferred from Figures 6 and 7 that Thirunavalur, Dindigul, Madurai, and Vidyut Nagar are reported to exhibit more water scarce situation than any of the districts of Karnataka with decreasing rainfall trends. Situation could be further analysed in the subsequent section, where decadal percentage departures from the Normal have been analysed for both states to get more vivid findings.

Table 6 also shows the nonparametric pattern of annual rainfall in Karnataka districts with declining patterns. Only the Karur district in Tamil Nadu experiences a decrease in rainfall of less than $1.0 \mathrm{~mm}$. There has been no decrease in rainfall of less than $0.5 \mathrm{~mm}$ in any of the districts. A study also suggests that Ariyalur, Namakkal, Ramanathapuram, Thoothukudi, and districts of Vellore and Cuddalore have the lowest rainfall increase intervals of less than $0.5 \mathrm{~mm}$, while Cuddalore and Nagapattinam have the highest rainfall increase intervals of $2-3 \mathrm{~mm}$. Rainfall is not increasing in any of the districts more than $3 \mathrm{~mm}$.

In Karnataka, the lowest rainfall decrease periods are less than $0.5 \mathrm{~mm}$ in Bellary and Koppal, while the highest rainfall decrease intervals are more than $5 \mathrm{~mm}$ in Dharwad, Hassan, and Chamarajanagar. According to trend study, the smallest increase rainfall interval is less than $0.5 \mathrm{~mm}$ in Bagalkot and Kolar, while the maximum increase rainfall interval is 1$2 \mathrm{~mm}$ in Bangalore, Bijapur, Gulbarga, and Kodagu.

5.1.3. Decadal Percentage Departure from the Normal. It is observed that epochal behaviour of rainfall is almost similar in character with equivalent numbers of wet and dry periods with decadal mean percentage departure from normal range with $+20 \%$ to $-10 \%$ till $1991-2000$.

However, the most recent decadal departure (for 2001-2010) is almost in the range of $-40 \%$ and higher with high frequency of deficient years in almost all meteorological district stations of both states. Departure of $-10 \%$ is reported in only 1981-1990 with all other decades in almost positive side of departure for all districts with deficient rainfalls in Karnataka and only in Nilgiri in Tamil Nadu. The normal or positive phases started from the year 1901 and almost continued till to the end of 1981 in case of Karnataka. 


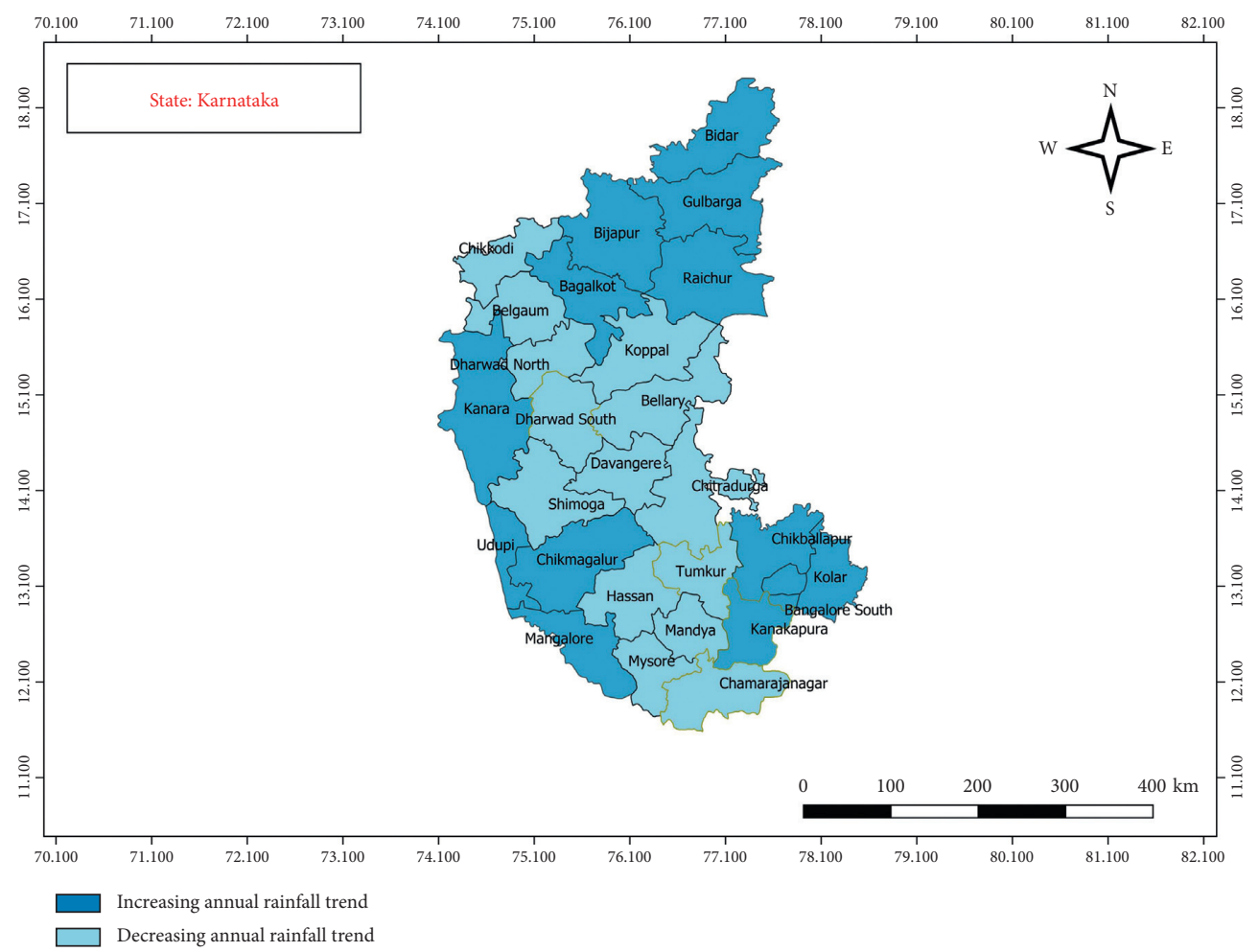

(a)

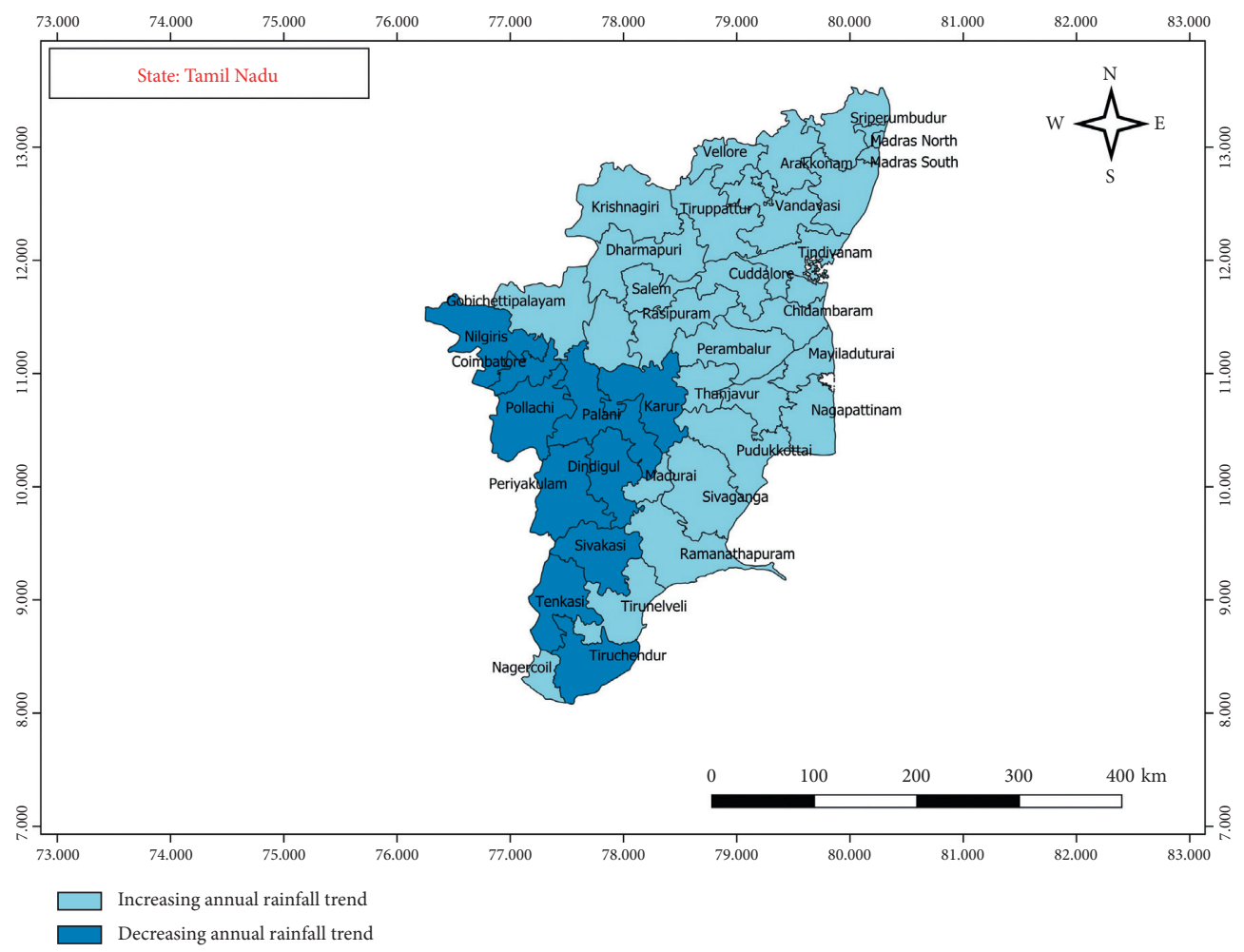

(b)

FIGURE 8: District-wise annual rainfall trend for both states.

The most recent alternate sequence of multidecadal periods having frequent droughts years are clearly noticed in Figures 9 and 10 for all deficient district of Karnataka with only one district, namely, Nilgiri in Tamil Nadu. Additionally, one can delineate dry periods: (i) 1921-1930 for Belgaum (Karnataka); (ii) 1931-50 for Nilgiri (Tamil Nadu); (iii) 1931-40 and 1961-70 


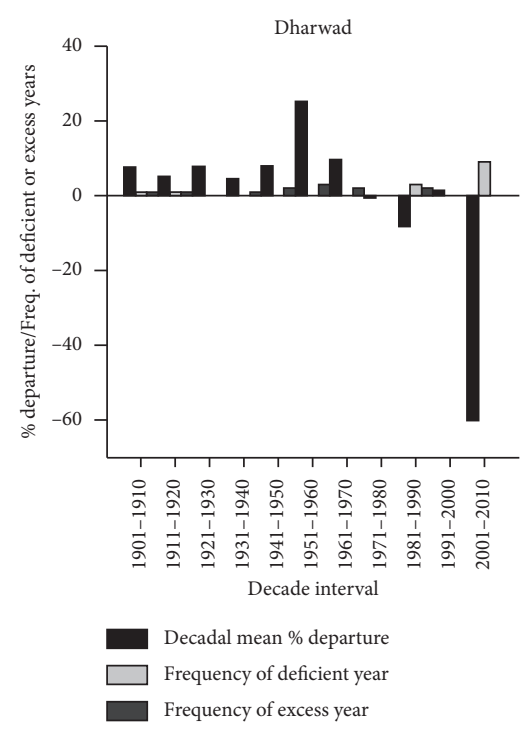

(a)

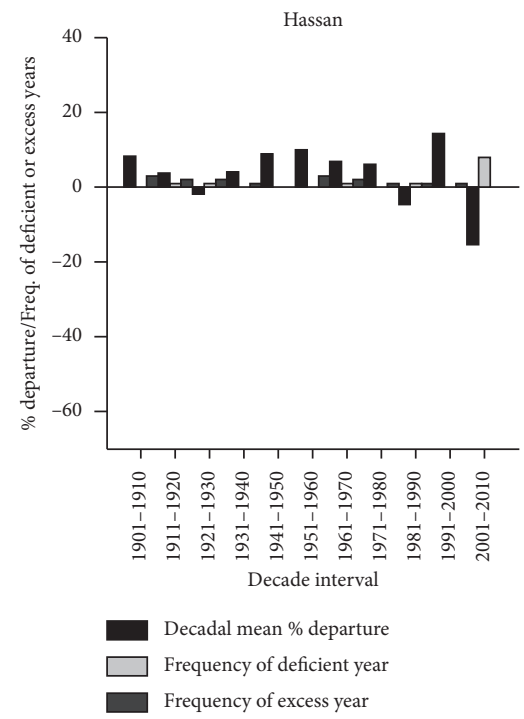

(d)

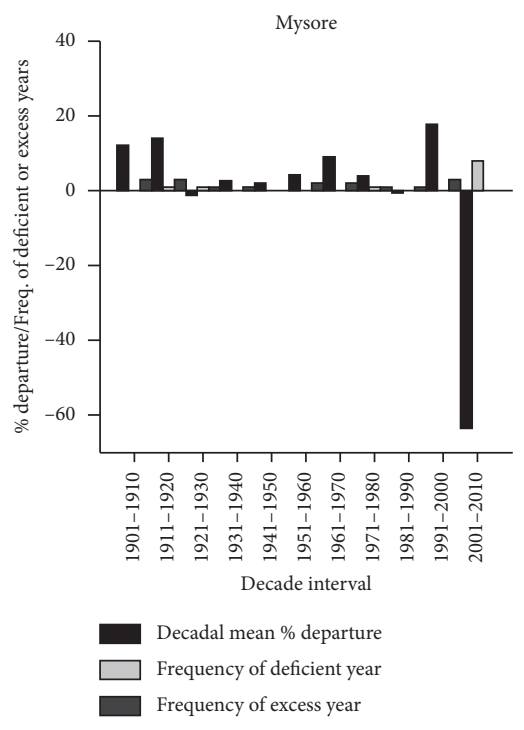

(b)

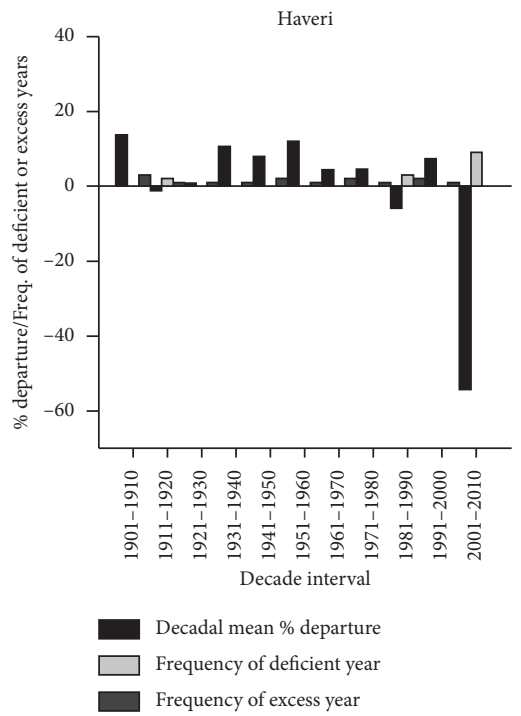

(e)

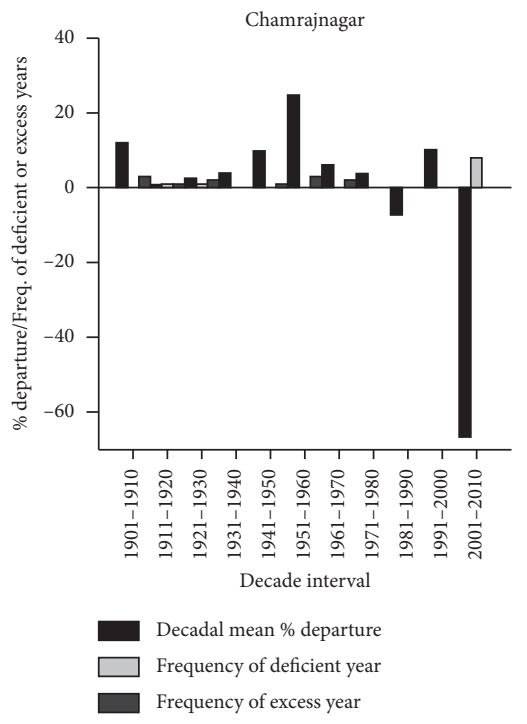

(c)

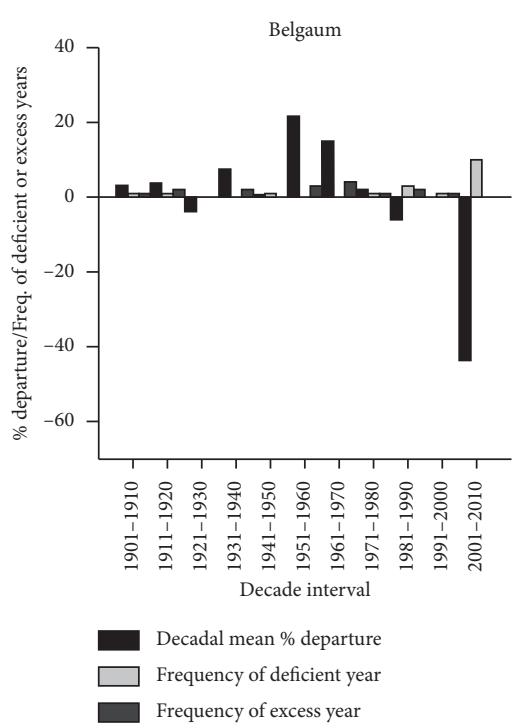

(f)

Figure 9: Decadal mean percentage departure from normal for Karnataka region.

for Vidyut Nagar (Tamil Nadu); (iv) 1921-1950 for Madurai (Tamil Nadu) (v) 1921-1930 and 1941-1950 for Dindigul (Tamil Nadu); (vi) 1931-1950 for Tirunelveli (Tamil Nadu).

\subsection{Drought}

5.2.1. Drought Index. The graphical comparison based on no drought, mild drought, moderate drought, and severe drought based on DI values for both states are vividly depicted in Figure 20 for illustration. It can be found that so far drought recurrence is concerned (mostly mild drought cases), and Tamil Nadu is predominating. However, in terms of severity (moderate or severe drought situation), Karnataka is ahead of Tamil Nadu. However, to get better insight another drought index, namely, Palmer drought index, as described in Section 2.3 was evaluated for all meteorological district stations for both study regions in subsequent section.
5.2.2. Palmer Drought Index. During the study period, it was observed that the incipient dry spell (IDS) condition was prevalent in almost all district gauge stations in Karnataka for a significant number of years. However, moderate-tosevere drought was observed in several districts, including Chamarajanagar, Dharwad, Belgaum, Haveri, and Mysore, for at least 5-10 years.

Apart from drought event years, climatic conditions, such as moderately wet (MW), near normal (NN), slightly wet (SW), and incipient wet spell (IWS), could be identified in different districts in an average of three event years. One event year with a very wet climate was identified in three districts, along with two other event years with a very wet climate (in Shimoga district).

In the case of Palmer drought index values, none of the districts showed signs of extremely wet (EW) climate conditions. Similarly, in case of Tamil Nadu, incipient dry 


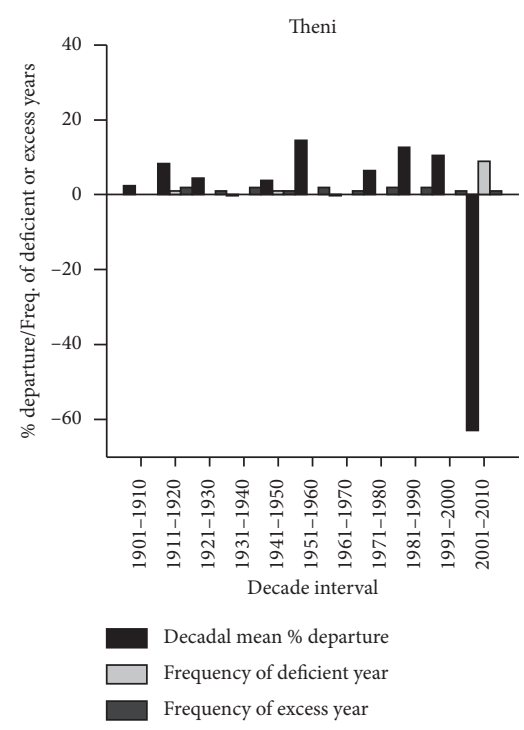

(a)

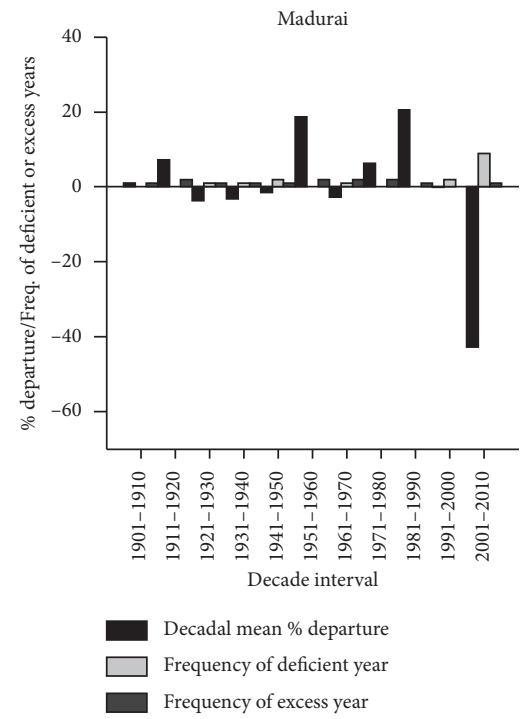

(d)

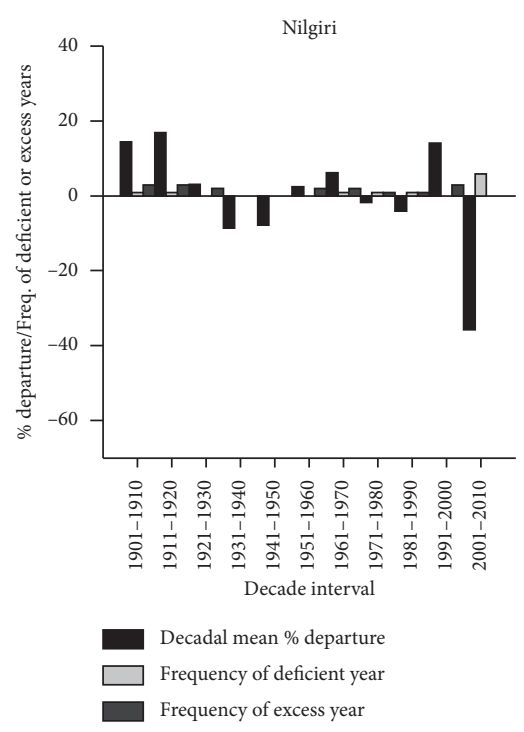

(b)

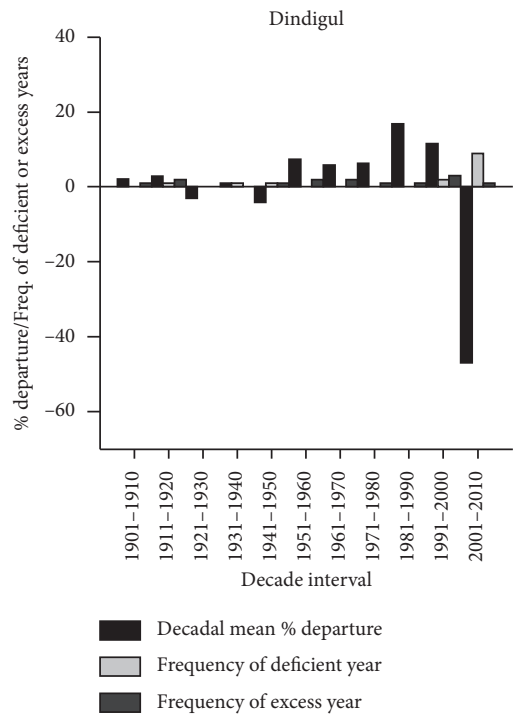

(e)

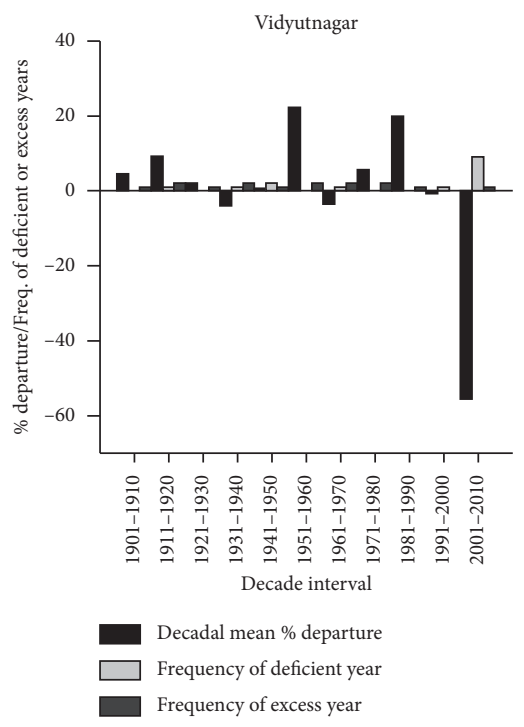

(c)

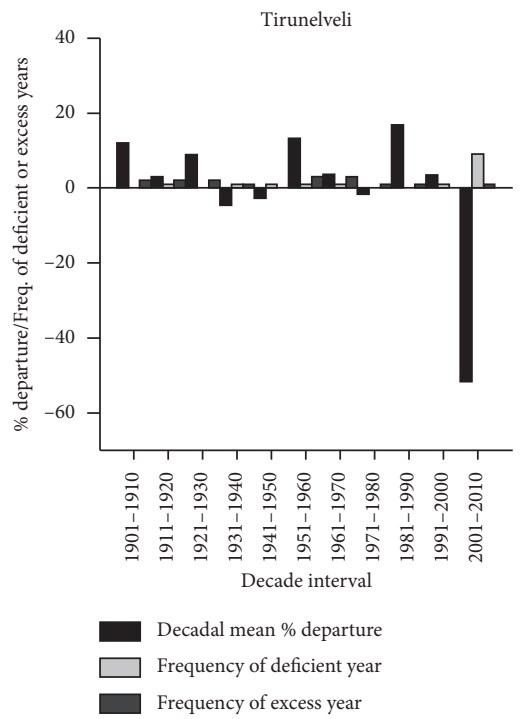

(f)

Figure 10: Decadal mean percentage departure from normal for Tamil Nadu region.

spell (IDS) was prevalent in all districts of Tamil Nadu. In at least 15-17 event years of mild-to-moderate drought, 18 event years of incipient dry spell were inferred in Tamil Nadu, mostly in districts of Nilgiri, Vidyut Nagar, Dindigul, and Theni in the past 110 years.

It is pertinent to mention that, for both states, almost identical event years (44 times) were under near normal (NN) condition with 12-13 event years as slightly wet and three event years as moderately wet conditions. Furthermore, not extremely wet or very wet conditions were found in any district of either state during the study period. According to the findings, mildto-moderate drought predominated (with higher event years) in Tamil Nadu, while moderate-to-severe drought occurred in Karnataka but for a shorter period (10-11 years).
5.2.3. Standardized Precipitation Index. One can observe that mean event years for moderately dry and severely dry were considerably higher in case of Tamil Nadu, whereas, for extremely dry conditions, Karnataka was registering a higher value.

Similarly, regarding statistical spread of the distribution, Tamil Nadu registered lower values than that of Karnataka. It means that higher numbers of event years were nearer to peak events values. It is also suggestive of relatively higher vulnerability of drought situations in Tamil Nadu.

In conclusion, Tamil Nadu had more vulnerability of moderate-to-severe drought situation with larger areal extend, whereas Karnataka had more localized drought vulnerability with severity on higher side. 


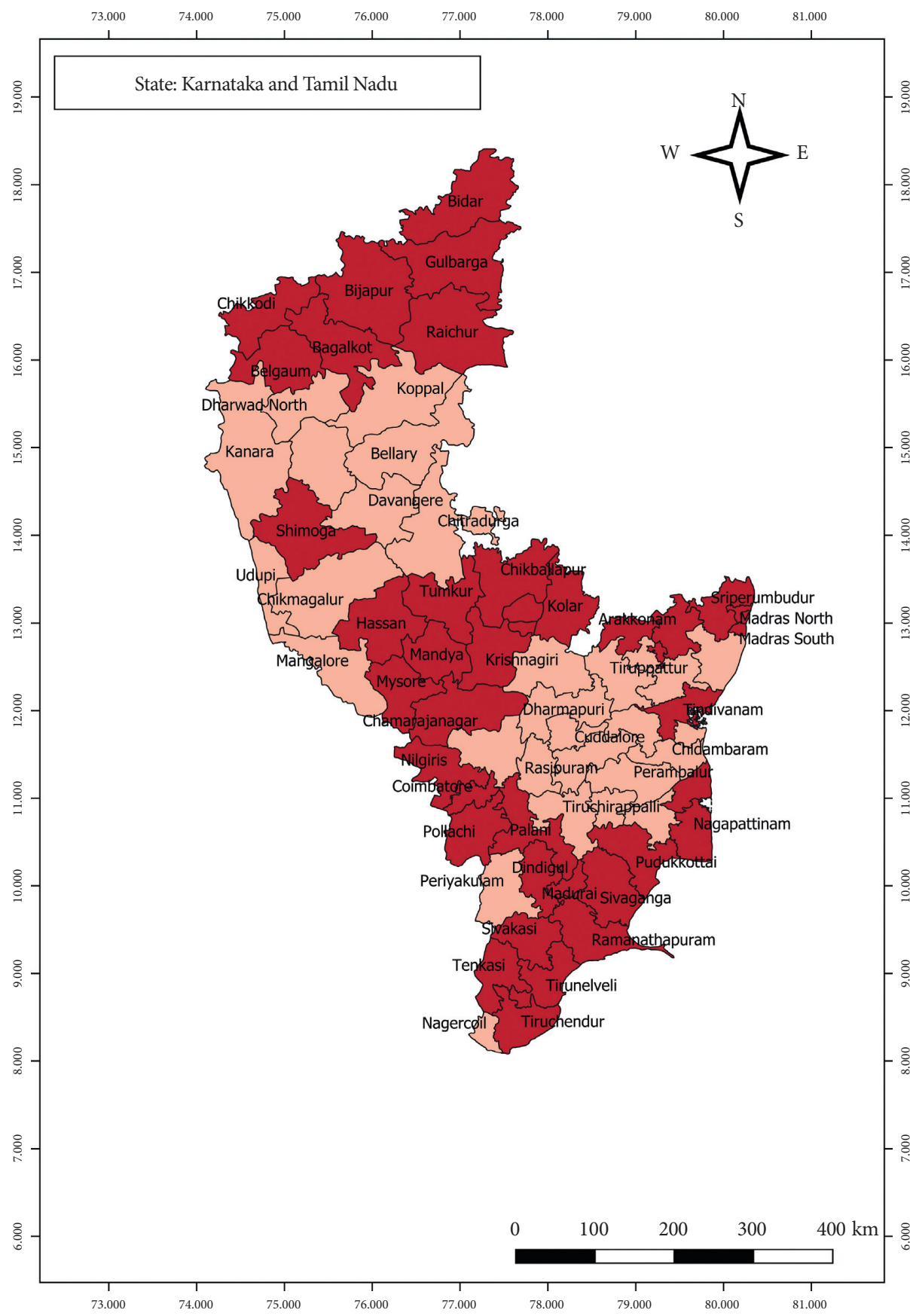

$\square$ Drought affected district

FIGURE 11: Drought affected districts of study region as per DI threshold values.

From the previously mentioned analysis for two regions, it can be concluded that drought characterization using three drought indicators, namely, DI, PDI, and SPI, produced somehow coherent information, but the use of PDI is limited and complex to assess with respect to DI and SPI, as it requires huge additional data and computations to perform an adequate and accurate soil water balance, while the DI and SPI needs only precipitation data, which are more easily available in numerous locations of the study areas.
It is also concluded that using SPI is more meaningful relative to DI as it incorporates seasonal variation also, and better statistical information can be obtained for further indepth analysis. Using PDI, however, is much less accurate for yearly forecasts [58]. The present work is inspired by numerous peer-reviewed published articles, where single variable has been used for estimation and statistical analysis [26-28, 31-34, 54, 59]. However, recent global challenges, such as climate change and global warming, have emphasized the need to continue working on better drought 
TABle 7: Number of drought event years with respect to SPI values for districts of Tamil Nadu and Karnataka.

\begin{tabular}{|c|c|c|c|c|c|c|c|c|}
\hline \multirow{2}{*}{ SL. No. } & \multicolumn{4}{|c|}{ Tamil Nadu } & \multicolumn{4}{|c|}{ Karnataka } \\
\hline & District & MD (years) & $\mathrm{SD}$ (years) & ED (years) & District & MD (years) & SD (years) & ED (years) \\
\hline 1 & Ariyalur & 12 & 5 & 0 & Bagalkot & 14 & 4 & 0 \\
\hline 2 & Chennai & 11 & 5 & 0 & Bangalore rural & 8 & 7 & 1 \\
\hline 3 & Coimbatore & 2 & 3 & 5 & Bangalore & 9 & 9 & 0 \\
\hline 4 & Cuddalore & 8 & 4 & 0 & Belgaum & 8 & 5 & 4 \\
\hline 5 & Dharmapuri & 17 & 3 & 1 & Bellary & 18 & 4 & 1 \\
\hline 6 & Dindigul & 12 & 6 & 3 & Bidar & 16 & 4 & 0 \\
\hline 7 & Erode & 0 & 0 & 0 & Bijapur & 14 & 4 & 1 \\
\hline 8 & Kancheepuram & 12 & 6 & 0 & Chamarajanagar & 1 & 2 & 6 \\
\hline 9 & Karur & 11 & 5 & 3 & Chitradurga & 11 & 7 & 1 \\
\hline 10 & Madurai & 10 & 7 & 1 & Devanagari & 12 & 3 & 4 \\
\hline 11 & Nagapattinam & 16 & 2 & 0 & Dharwad & 4 & 2 & 7 \\
\hline 12 & Namakkal & 13 & 5 & 0 & Gadag & 11 & 5 & 3 \\
\hline 13 & The Nilgiris & 10 & 2 & 0 & Gulbarga & 14 & 5 & 2 \\
\hline 14 & Perambalur & 16 & 5 & 0 & Hassan & 4 & 3 & 5 \\
\hline 15 & Pudukkottai & 9 & 5 & 2 & Haveri & 7 & 1 & 7 \\
\hline 16 & Ramanathapuram & 11 & 8 & 1 & Kodagu & 12 & 2 & 0 \\
\hline 17 & Saleem & 16 & 3 & 1 & Kolar & 9 & 6 & 1 \\
\hline 18 & Shivaganga & 11 & 7 & 2 & Koppal & 14 & 4 & 2 \\
\hline 19 & Thanjavur & 16 & 3 & 1 & Mandya & 5 & 2 & 4 \\
\hline 20 & Theni & 3 & 1 & 7 & Mysore & 0 & 0 & 0 \\
\hline 21 & Thiruvallur & 5 & 6 & 0 & Raichur & 10 & 3 & 1 \\
\hline 22 & Tiruchirappalli & 9 & 5 & 1 & Shimoga & 12 & 3 & 1 \\
\hline 23 & Tirunelveli & 15 & 2 & 3 & Tumkur & 11 & 5 & 2 \\
\hline 24 & Tiruvannamalai & 15 & 5 & 1 & & & & \\
\hline 25 & Thoothukudi & 14 & 6 & 1 & & & & \\
\hline 26 & Vellore & 9 & 5 & 1 & & & & \\
\hline 27 & Viluppuram & 13 & 5 & 1 & & & & \\
\hline 28 & Virudhunagar & 12 & 3 & 2 & & & & \\
\hline Mean & & 11 & 4.36 & 1.32 & & 9.74 & 3.91 & 2.30 \\
\hline Standard & deviation & 4.28 & 1.87 & 1.63 & & 4.54 & 2.04 & 2.21 \\
\hline
\end{tabular}

Source: [49].

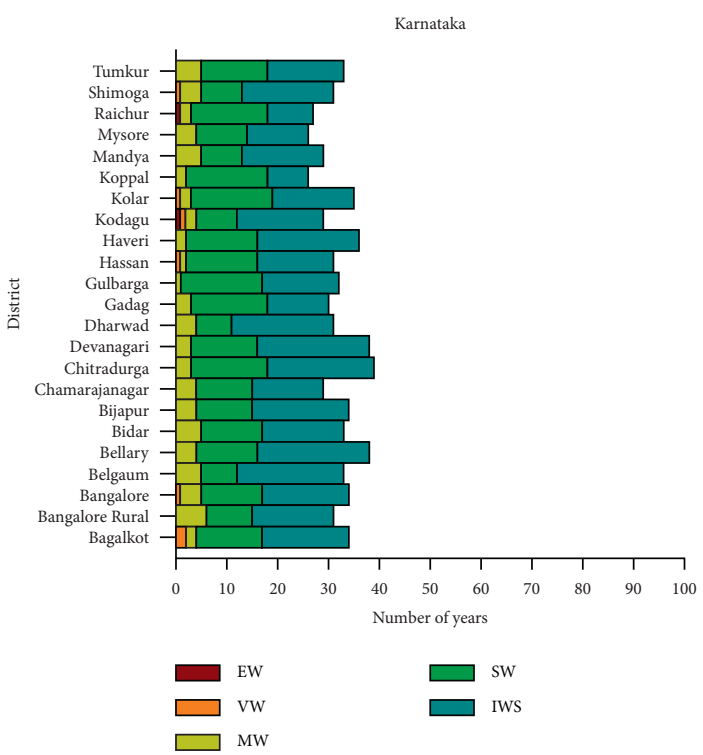

(a)

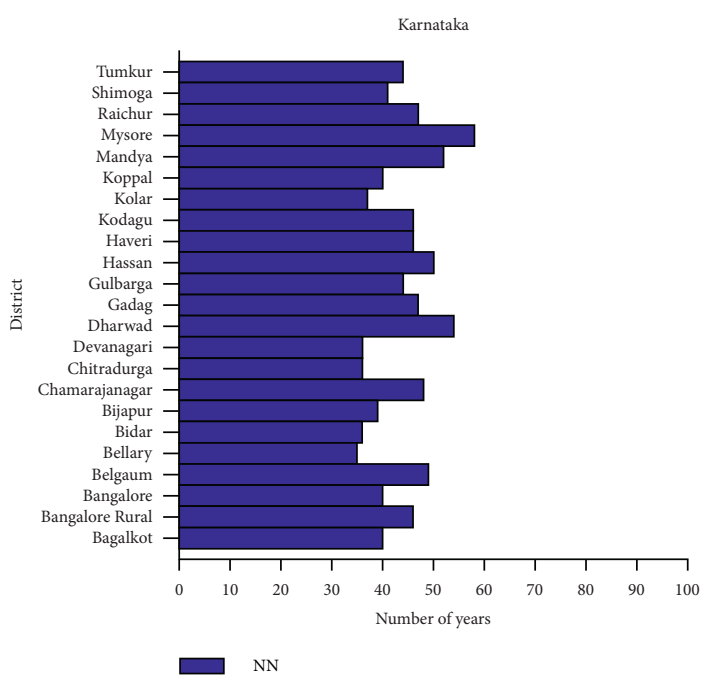

(b)

FIgURE 12: Continued. 


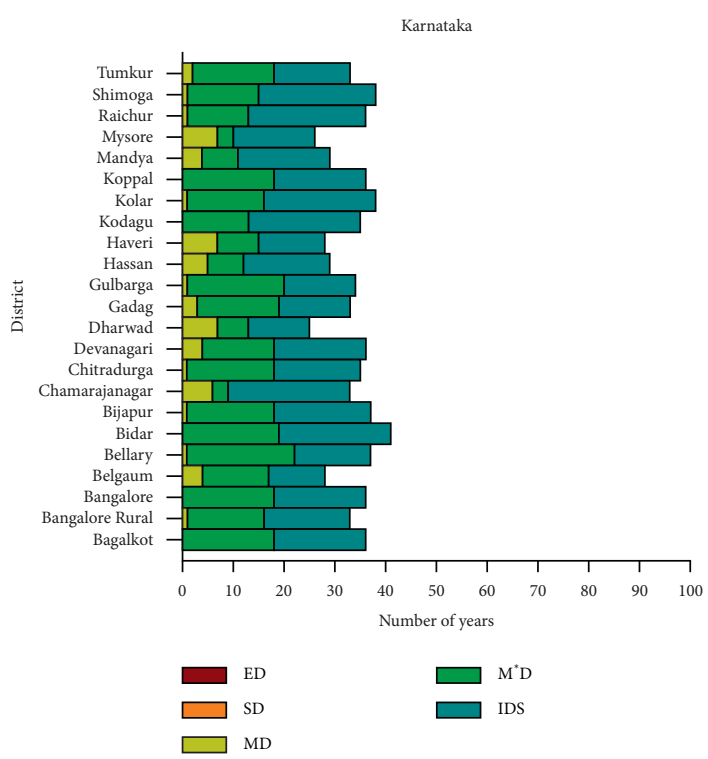

(c)

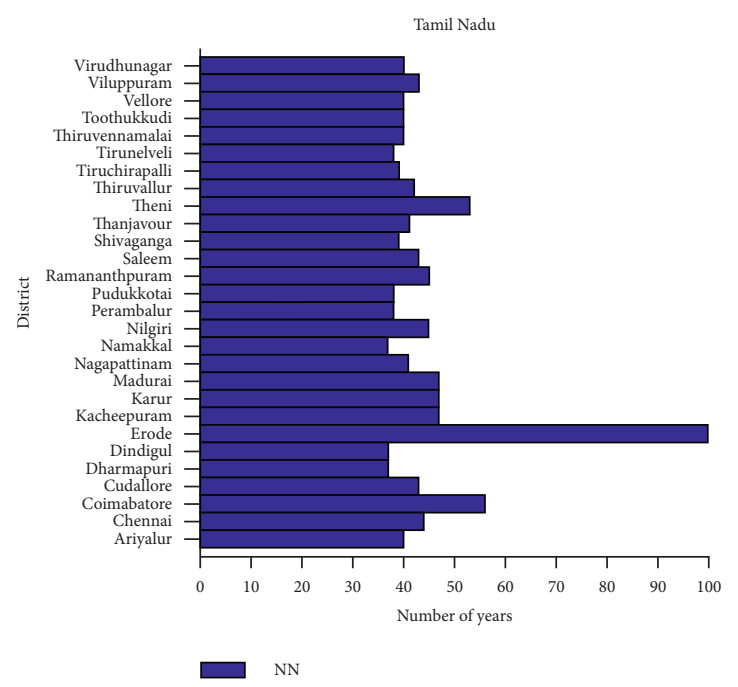

(e)

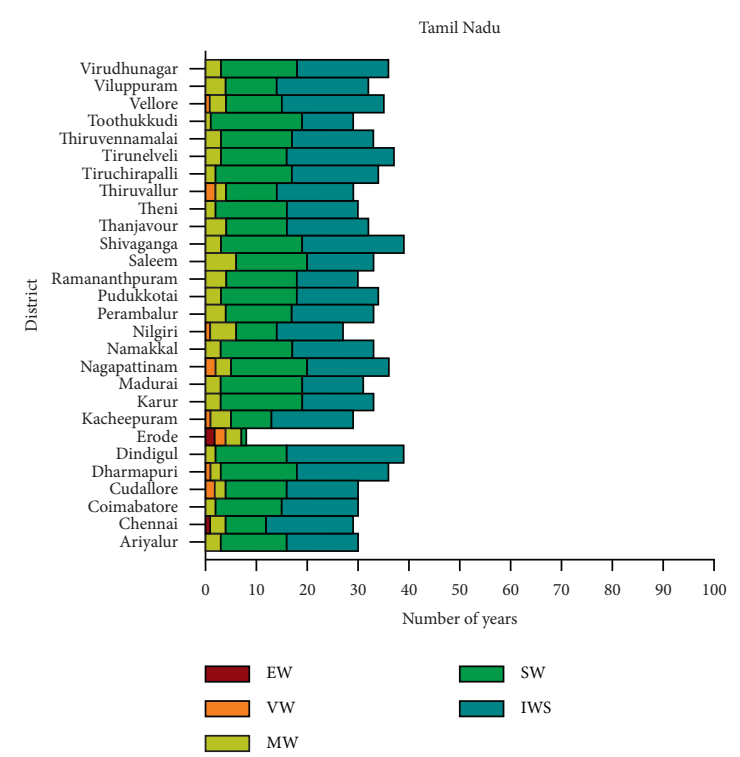

(d)

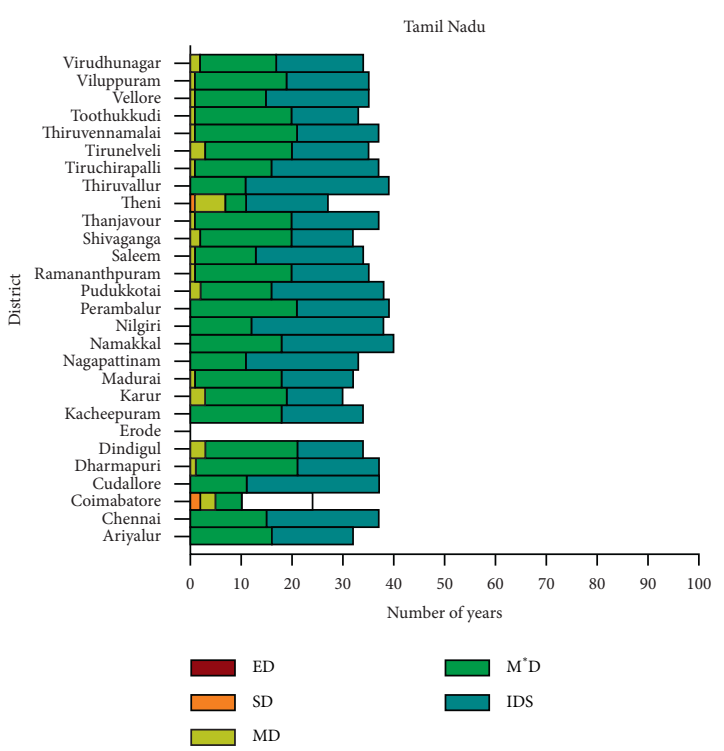

(f)

FIGURE 12: Drought intensity based on SPI threshold values.

indicators and approaches with adequate incorporation of multiple climatic variables [60]. A single variable-based estimation may likely to underestimate drought situation [61]. To sum up, proper and adequate integration of all relevant data and drought definitions with respect to the most common forms of monthly droughts in time and space, as well as climate change scenarios, should be addressed [61]. Since practically all these indices, including the study reported in this paper, are based on observed data as key performance indicators, drought indices may not produce universally agreed conclusions [58]. Drought risk and their implications have become a contentious issue among scientists, with debates continuing to occur around the world. A better understanding is required on the various indices, which are proposed until now with adequate incorporation of significant parameters influencing climate changes in a region.

5.2.4. Drought Vulnerability Assessment for Karnataka. Based on statistical approach (Figure 13), Dharwad district has the highest percentage probability of drought exceedance, followed by Belgaum district under moderate drought conditions. However, the Kodagu district has the lowest probability of exceedance at 2.3 percent.

Similarly, Dharwad district has again the highest probability of exceedance, followed by Mysore, Bangalore, and Bagalkot for severe drought conditions. 


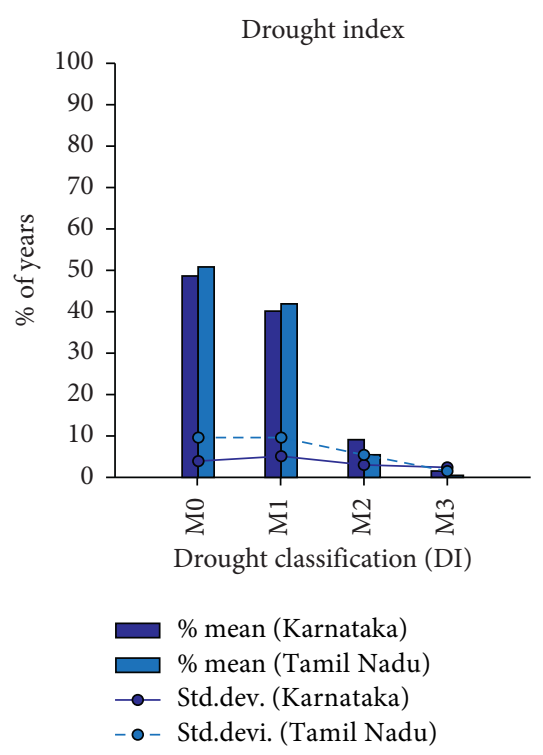

(a)

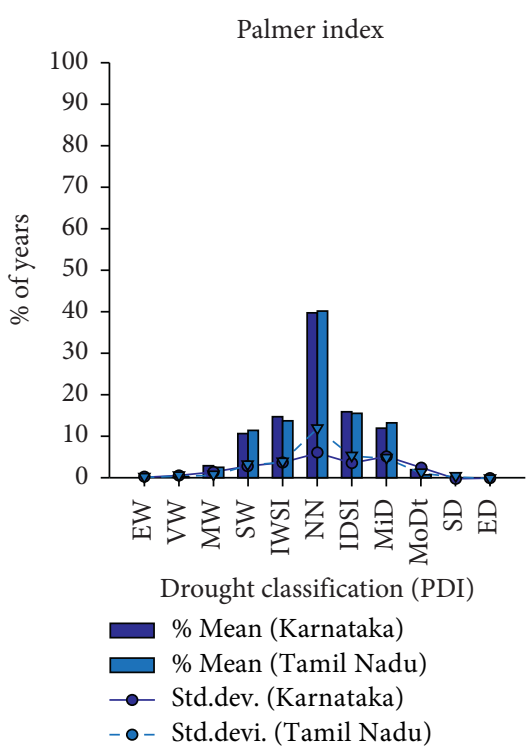

(b)

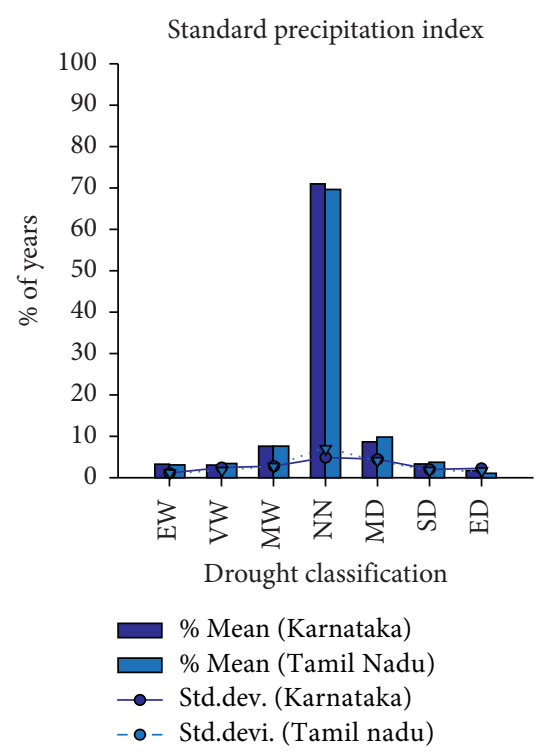

(c)

FIGURE 13: Yearly drought recurrence for 110 years based on three chosen drought indices.

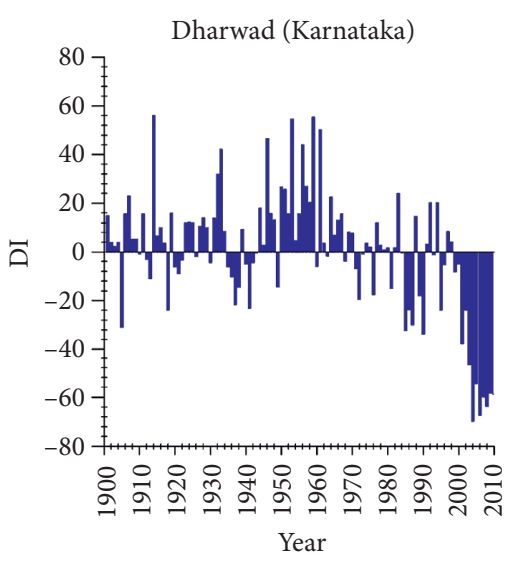

DI

(a)

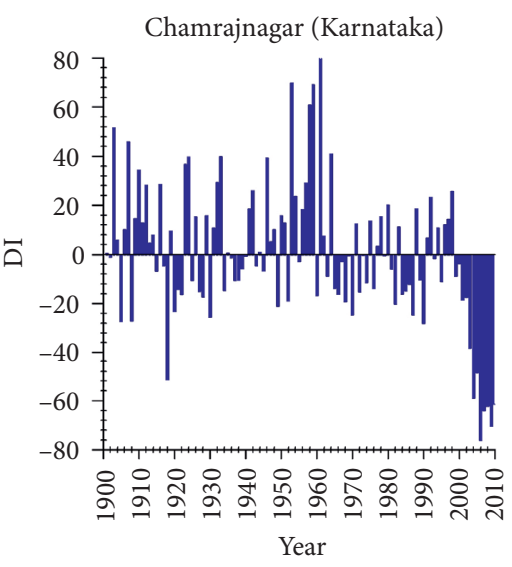

DI

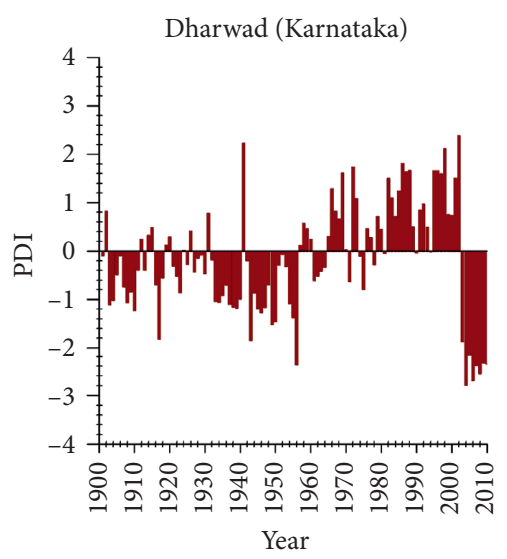

(b)

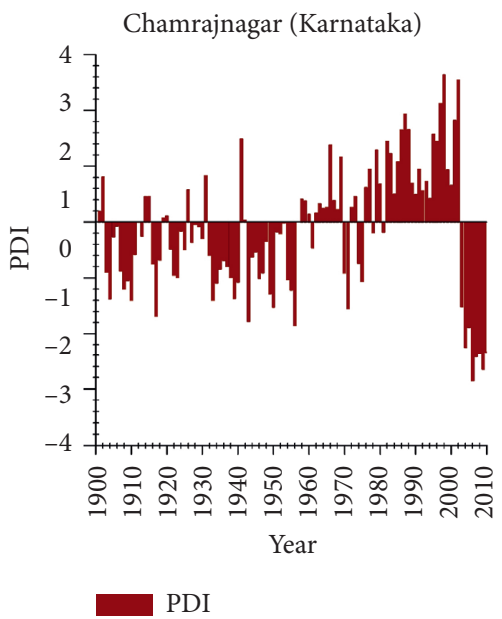

(e)

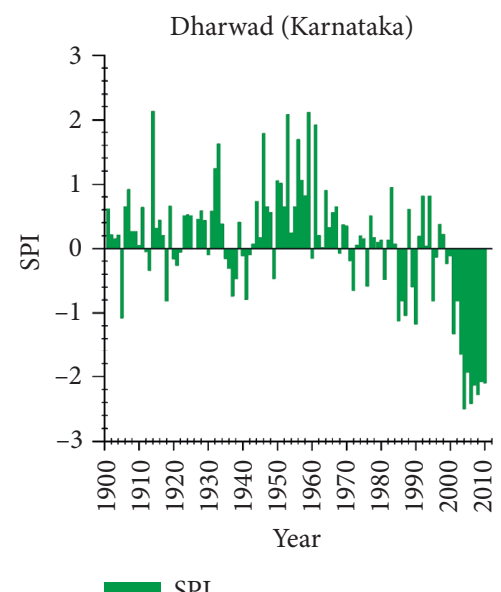

(c)

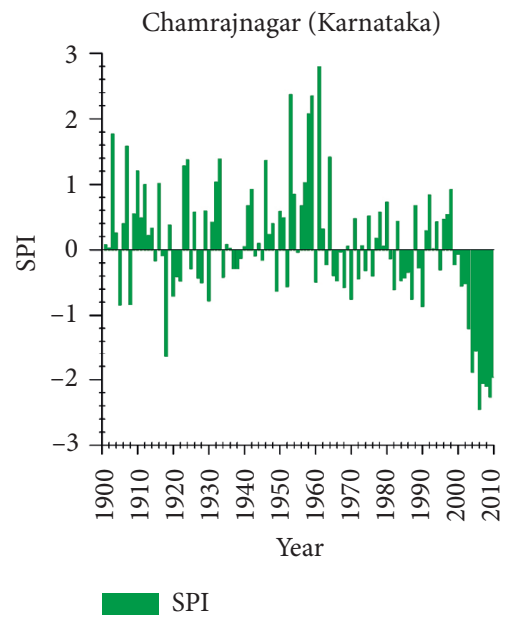

(f)

Figure 14: Yearly variation of computed chosen drought indices for two chosen districts of Karnataka. 


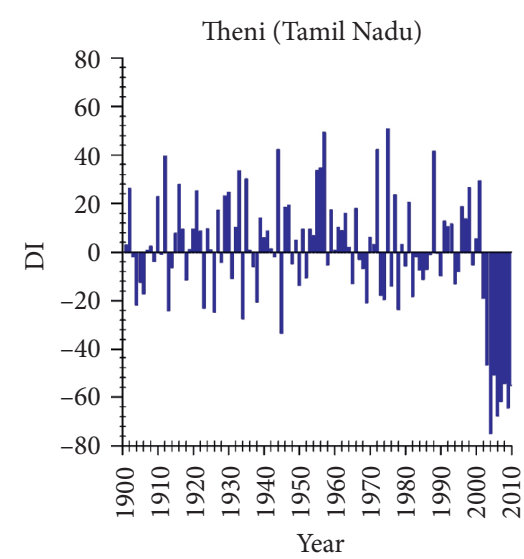

$\square$ DI

(a)

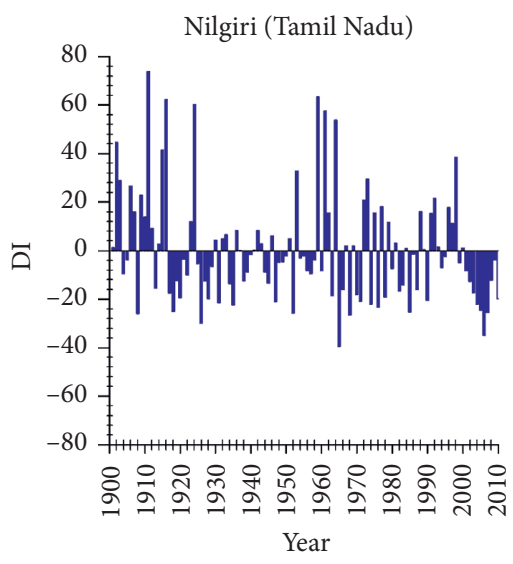

(d)

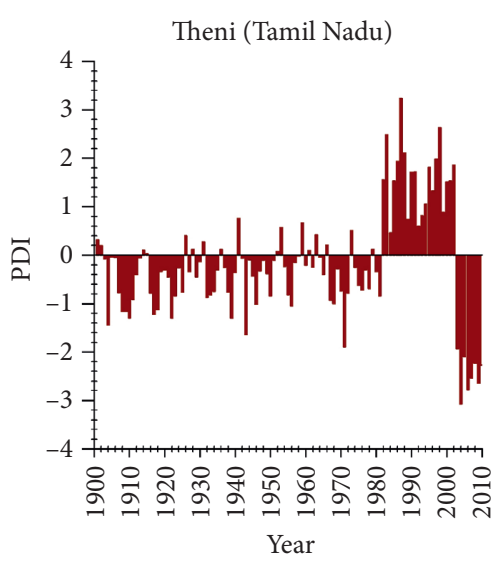

PDI

(b)

Nilgiri (Tamil Nadu)

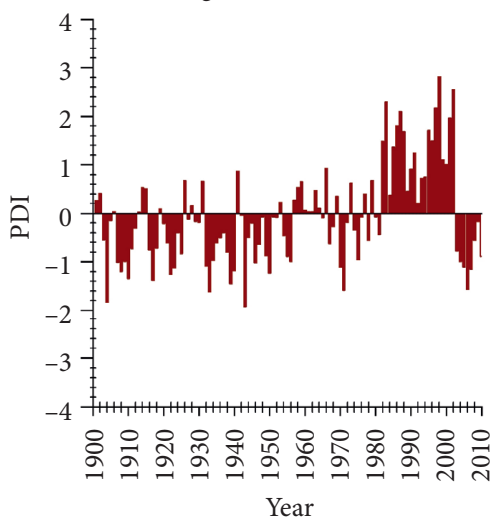

PDI

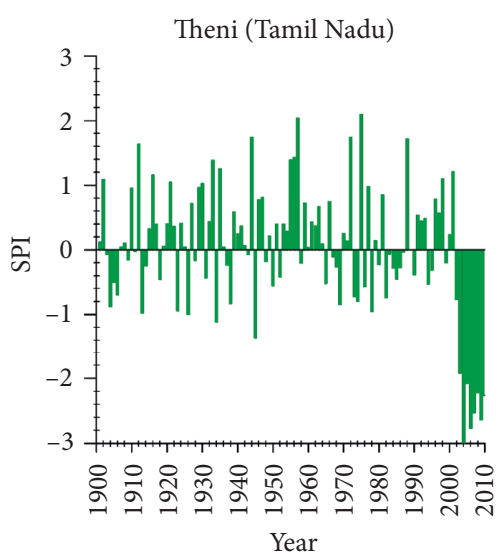

SPI

(c)

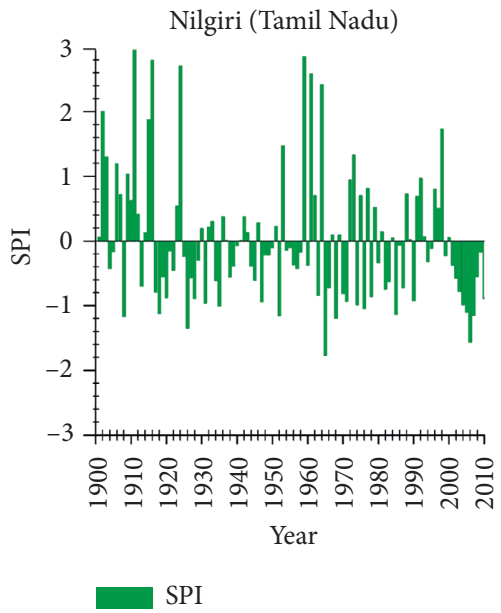

(f)

FIGURE 15: Yearly variation of computed chosen drought indices for two chosen districts of Tamil Nadu.

TABLE 8: Threshold values of scaled SPI (drought deficit).

\begin{tabular}{lccccccc}
\hline Classification & EW & VW & MW & NN & MD & SD & ED \\
\hline Scaled SPI value & $\geq 7$ & 6.5 to 6.99 & 6 to 6.49 & 4.01 to 5.99 & 4.0 to 3.51 & 3.5 to -3.01 & $\leq 3$ \\
\hline
\end{tabular}

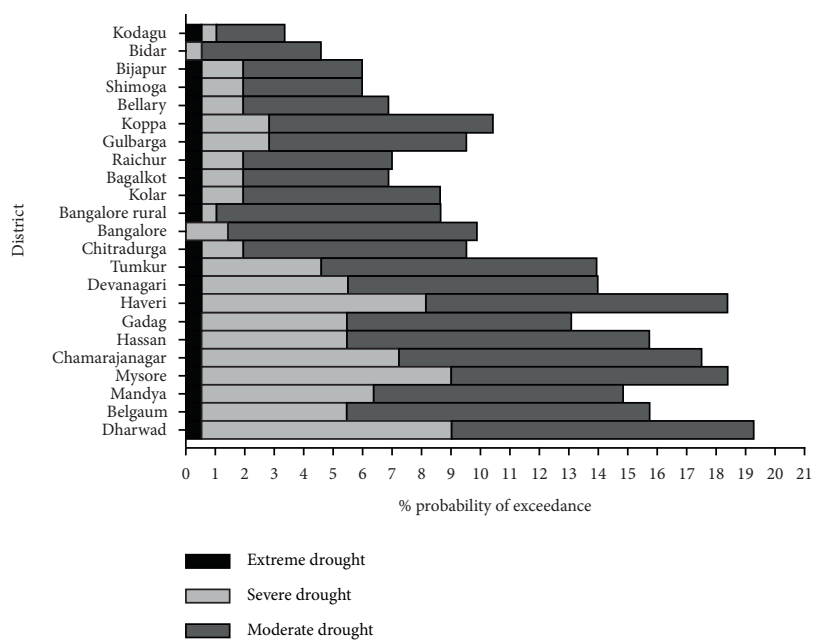

FIGURE 16: Percentage exceedance probabilities for drought events using analytical LPIII technique for 110 years on with scaled SPI values for Karnataka. 


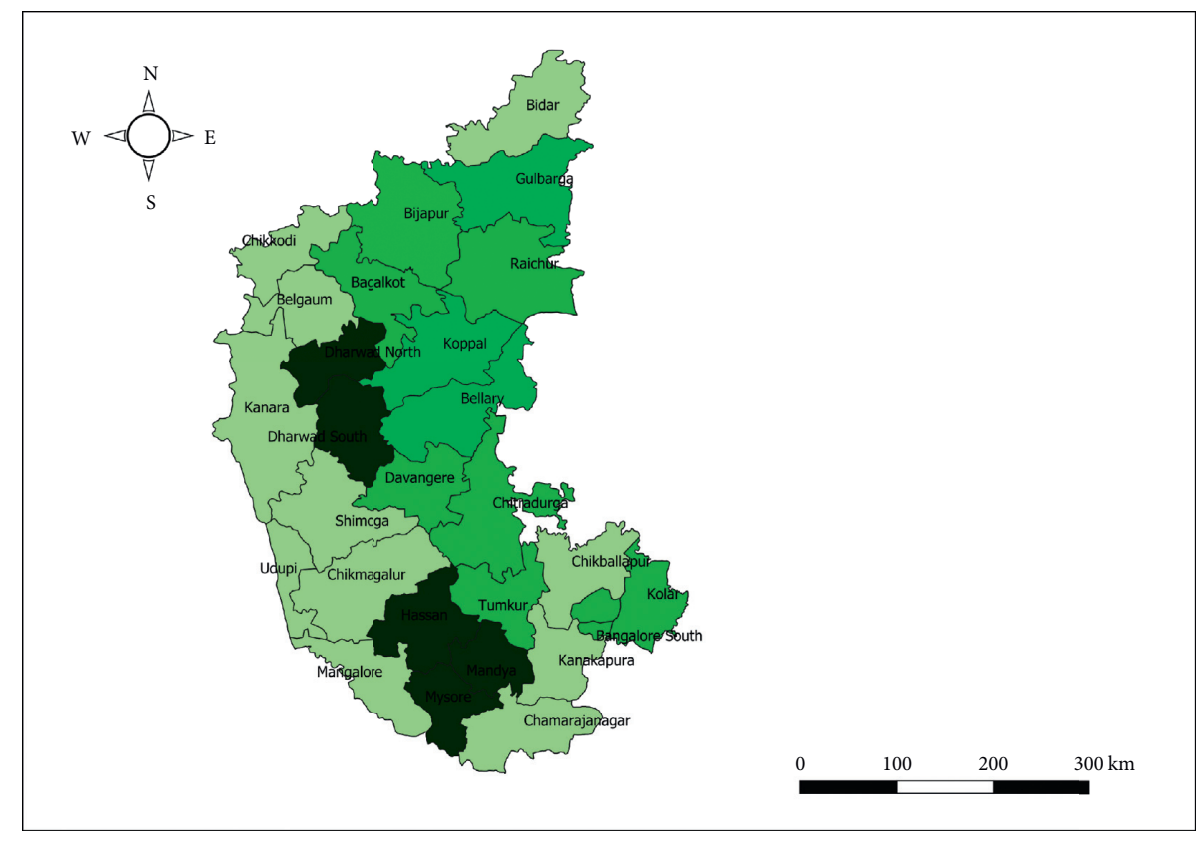

Return period (years)

$\begin{array}{lr}3-10 & 22-44 \\ 11-21 & 45-51\end{array}$

FIgURE 17: Vulnerability map of moderate drought (MD) ranges for Karnataka region.

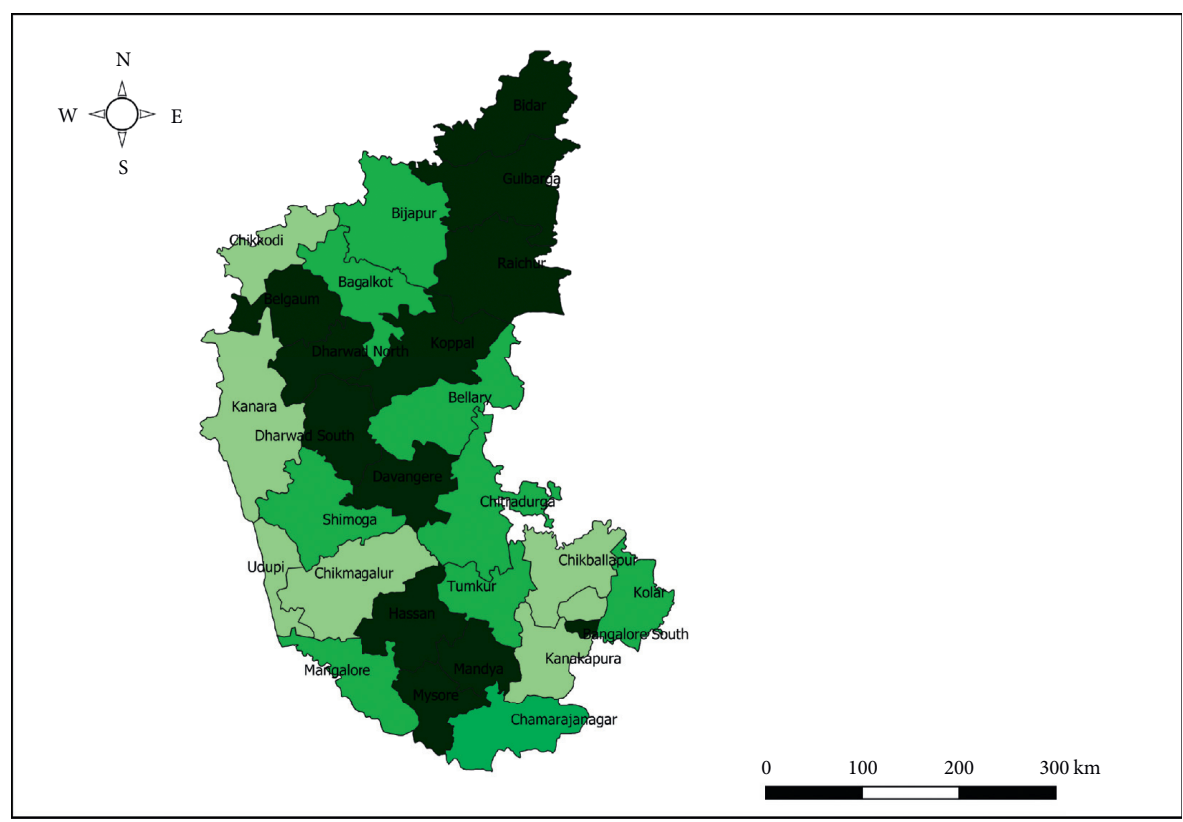

Return period (years)

$5-250$

$251-800$

$900-1600$

$1700-2500$

FIGURE 18: Vulnerability map of severe drought (SD) ranges for Karnataka region.

In the event of extreme drought, Dharwad district is found to have the highest risk of exceeding the drought threshold, followed by Gulbarga district. Mandya, Gadag, Devanagari, Tumkur, Bangalore, Raichur, and Bider, however, were found to have the lowest exceedance probabilities.
Using GIS tool, corresponding vulnerability maps for moderate drought, severe drought, and extreme drought are presented in Figures 17-19 for illustration. Relative vulnerability as per threshold drought deficit (Table 8) is vividly depicted. 


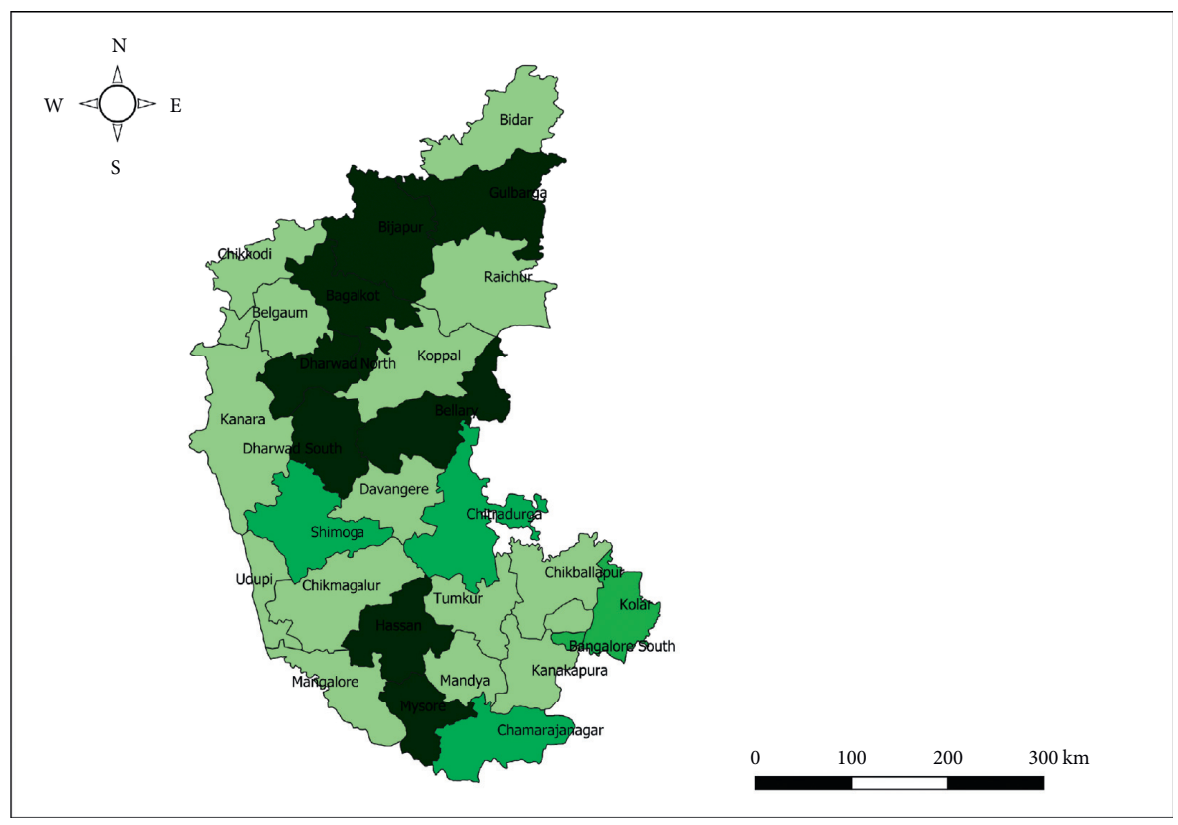

Return period (years)

65-1000

$2100-9000$

$1100-2000$

Figure 19: Vulnerability map of extreme drought (ED) ranges for Karnataka region.

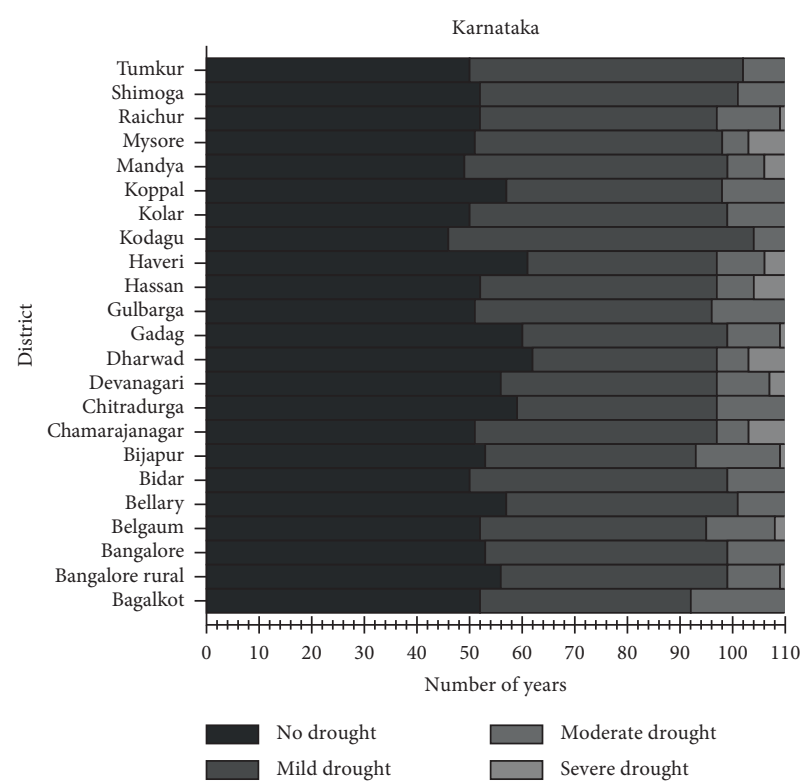

(a)

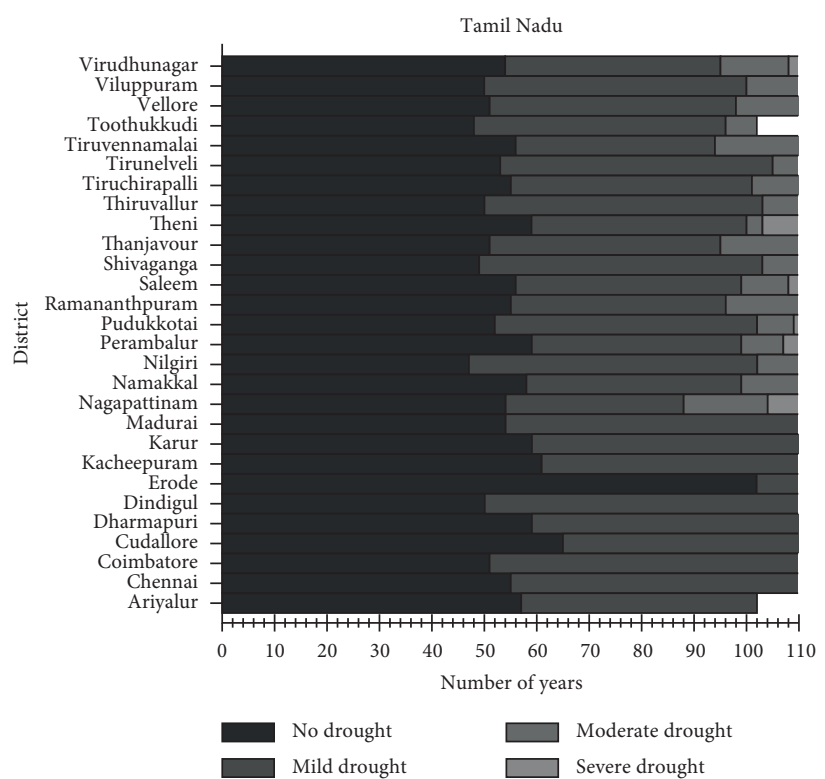

(b)

FIGURE 20: Drought intensity based on DI values.

\section{Conclusion}

(1) The current study compared two southern Indian states with characterisation of their precipitation for 110 years of historical rainfall data.

(2) Drought characterization using three drought indicators, namely, DI, PDI, and SPI, produces coherent information. It is also concluded that using SPI is more meaningful relative to DI as it incorporates seasonal variation also, and better statistical information can be obtained for further in-depth analysis. However, it is also concluded that single variablebased estimation of drought intensity may likely to underestimate the drought risk and its implications. 
(3) Tamil Nadu was found to be more vulnerable to moderate-to-severe drought situation with larger areal extend, whereas Karnataka had more localized drought vulnerability with severity on higher side.

(4) The monotonic trend of rainfall in Karnataka and Tamil Nadu regions over the last 110 years was investigated using nonparametric methods, such as Mann-Kendall and Sen's slope tests. It was found that decreasing trend in Karnataka is more significant compared with that of Tamil Nadu.

(5) Decadal mean \% departure from the normal sufficiently depicts that drought vulnerability in both states has triggered in the most recent decade (2001-2010), compared with previous decades.

(6) Log Pearson probability distribution technique using SPI is attempted to assess exceedance probabilities of meteorological drought condition of classified thresholds for Karnataka region. It was found that Dharwad district in Karnataka is the most vulnerable among 23 districts, with respect to severity and recurrence of drought conditions.

\section{Data Availability}

The data for this analysis were collected from the India Water Portal (https://www.indiawaterportal.org/met_data), Bhuvan (http://bhuvan.nrsc.gov.in/bhuvan_links.php\#), and the Indian Meteorological Department.

\section{Conflicts of Interest}

The authors declare no conflicts of interest.

\section{Acknowledgments}

The authors gratefully acknowledge the India Water Portal, Bhuvan (National Remote Sensing Centre), Government of India, and the Indian Meteorological Department for providing the data for analysis.

\section{References}

[1] J. S. Samra, Review and Analysis of Drought Monitoring Declaration and Management in India, International Water Management Institute, Battaramulla, Sri Lanka, 2004.

[2] D. A. Mooley, B. Parthasarathy, and A. A. Munot, "Large scale drought over India and their impact on agricultural production," Mausam, vol. 35, p. 265, 1984.

[3] A. Araghi, C. J. Martinez, J. Adamowski, and J. E. Olesen, "Spatiotemporal variations of aridity in Iran using highresolution gridded data," International Journal of Climatology, vol. 38, pp. 2701-2717, 2018.

[4] U. S. De, R. K. Dube, and G. S. Prakasa Rao, "Extreme weather events over India in last 100 years," Journal of the Indian Geophysical Union, vol. 9, no. 3, pp. 173-187, 2005.

[5] V. U. Smakhtin and D. A. Hughes, "Automated estimation and analyses of meteorological drought characteristics from monthly rainfall data," Environmental Modelling \& Software, vol. 22, pp. 880-890, 2007.
[6] D. S. Pai, L. Sridhar, P. Guhathakurta, and H. R. Hatwar, "District-wide drought climatology of the southwest monsoon season over India based on standardized precipitation index (SPI)," Natural Hazard, vol. 59, no. 3, pp. 1797-1813, 2011.

[7] F. Yusof, F. Hui-Mean, J. Suhaila, and Z. Yousof, "Characterisation of drought properties with bivariate copula analysis," Water Resources Management, vol. 27, no. 12, pp. 4183-4207, 2013.

[8] R. Shah, N. Bharadiya, and V. Manekar, "Drought index computation using standardized precipitation index (SPI) method for Surat district Gujarat," Aquatic Procedia, vol. 4, pp. 1243-1249, 2015.

[9] P. Wichitarapongsakun, C. Sarin, P. Klomjek, and S. Chuenchooklinb, "Rainfall prediction and meteorological drought analysis in the Sakae Krang river basin of Thailand," Agricultural and Natural Resources, vol. 50, pp. 490-498, 2016.

[10] P. K. Langat, L. Kumar, and R. Koech, "Temporal variability and trends of rainfall and streamflow in Tana river Basin Kenya," Sustainability, vol. 9, no. 1963, pp. 1-18, 2017.

[11] R. M. Hirsch, J. R. Slack, and R. A. Smith, "Techniques of trend analysis for monthly water quality data," Water Resources Research, vol. 18, pp. 107-121, 1982.

[12] S. Coles, An Introduction to Statistical Modeling of Extreme Values, Springer, Berlin, Germany, 2001.

[13] S. Yue, P. Pilon, and G. Cavadias, "Power of the Man$\mathrm{n}$-Kendall and Spearman's rho tests for detecting monotonic trends in hydrological series," Journal of Hydrology, vol. 259, pp. 254-271, 2002.

[14] S. Yue and M. Hashino, "Long term trends of annual and monthly precipitation in Japan," Journal American Water Resources Association, vol. 39, pp. 587-596, 2003.

[15] R. Modarres and V. D. P. R. da Silva, "Rainfall trends in arid and semi-arid regions of Iran," Journal of Arid Environment, vol. 70, pp. 344-355, 2007.

[16] A. Perera and U. Rathnayake, "Rainfall and atmospheric temperature against the other climatic factors: a case study from Colombo Sri Lanka," Mathematical Problems in Engineering, vol. 2019, Article ID 5692753, 15 pages, 2019.

[17] A. Girdhar, H. Kapur, V. Kumar, M. Kaur, D. Singh, and R. Damasevicius, "Effect of COVID-19 outbreak on urban health and environment," Air Quality, Atmosphere \& Health, vol. 14, pp. 389-397, 2021.

[18] H. Pannu, D. Singh, and A. Kaur Malhi, "Multi-objective particle swarm optimization-based adaptive neuro-fuzzy inference system for benzene monitoring," Neural Computing and Applications, vol. 31, no. 7, pp. 2195-2205, 2019.

[19] H. S. Pannu, D. Singh, and A. K. Malhi, "Improved particle swarm optimization based adaptive neuro-fuzzy inference system for benzene detection," CLEAN-Soil, Air, Water, vol. 46, no. 5, Article ID 1700162, 2018.

[20] S. Ghosh, P. Shivakumara, P. Roy, U. Pal, and T. Lu, "Graphology based handwritten character analysis for human behaviour identification," CAAI Transactions on Intelligence Technology, vol. 5, no. 1, pp. 55-65, 2020.

[21] B. Gupta, M. Tiwari, and S. S. Lamba, "Visibility improvement and mass segmentation of mammogram images using quantile separated histogram equalisation with local contrast enhancement," CAAI Transactions on Intelligence Technology, vol. 4, no. 2, pp. 73-79, 2019.

[22] H. S. Basavegowda and G. Dagnew, "Deep learning approach for microarray cancer data classification," CAAI Transactions on Intelligence Technology, vol. 5, no. 1, pp. 22-33, 2020.

[23] W. C. Palmer, Meteorological Drought, US Department of Commerce Weather Bureau, Silver Spring, MD, USA, 1965. 
[24] W. J. Gibbs and J. V. Maher, Rainfall Deciles as Drought Indicators, Bureau of Meteorology, Melbourne, Australia, 1967.

[25] T. B. McKee, N. J. Doesken, and J. Kliest, "The relationship of drought frequency and duration to time scales," in Proceedings of the 8th Conference of Applied Climatology, pp. 179-184, Anaheim CA American Meteorological Society, Boston MA, USA, January 1993.

[26] G. M. L. D. Quenum, N. A. B. Klutse, and D. Dieng, "Identification of potential drought areas in West Africa under climate change and variability," Earth System Environment, vol. 3, pp. 429-444, 2019.

[27] H. Al Adaileh, M. Al Qinna, and K. Barta, "A drought adaptation management system for groundwater resources based on combined drought index and vulnerability analysis," Earth System Environment, vol. 3, pp. 445-461, 2019.

[28] A. Mustafa and G. Rahman, "Assessing the spatio-temporal variability of meteorological drought in Jordan," Earth System Environment, vol. 2, pp. 247-264, 2018.

[29] A. Kumar, R. P. Pandey, and S. K Mishra, "Characteristics of meteorological droughts in north western India," Natural Hazards, vol. 94, no. 2, pp. 561-582, 2018.

[30] G. Mallya, V. Mishra, N. Dev, S. Tripathi, and S. Govindaraju Rao, "Trends and variability of droughts over the Indian monsoon region," Weather and Climate Extremes, vol. 12, pp. 43-68, 2016.

[31] L. Hangshing and P. P. Dabral, "Characterisation, trend assessment and copula based bivariate modelling of meteorological drought for central Brahmaputra valley-an agro climatic zone of Assam," Journal of Indian Water Resources. Society, vol. 38, no. 1, pp. 29-38, 2018.

[32] P. G. Oguntunde, B. J. Abiodun, and G. Lischeid, "Impacts of climate change on hydro-meteorological drought over the Volta Basin," West Africa Global Planet Change, vol. 155, pp. 121-132, 2017.

[33] P. G. Oguntunde, G. Lischeid, and B. J. Abiodun, "Impacts of climate variability and change on drought characteristics in the Niger River Basin," Stochastic Environment Research Risk Assessment West Africa, vol. 36, pp. 1017-1034, 2017.

[34] J. T. Al-Bakri, M. J. Alnaimat, E. Al-Karablieh, and E. A. Qaryouti, "Assessment of combined drought index and mapping of drought vulnerability in Jordan," International Journal of Engine Research Application, vol. 9, no. 3, pp. 5968, 2019.

[35] R. I. Gebert, The Cauvery River Dispute: Hydrological Politics in Indian Federalism, The University of British Columbia, Vancouver, Canada, 1983.

[36] N. Pani, "Institutions that cannot manage change: a gandhian perspective on the Cauvery dispute," Water Alternatives, vol. 2, no. 3, pp. 315-327, 2009.

[37] H. Mann, "Non-parametric tests against trend," Econometrica, vol. 13, pp. 245-259, 1945.

[38] M. Kendall, Rank Correlation Methods, Charles Griffin, London, UK, 4th edition, 1975.

[39] L. V. Alexander, X. Zhang, T. C. Peterson et al., "Global observed changes in daily climate extremes of temperature and precipitation," Journal of Geophysical Research: Atmospheres, vol. 111, pp. 105-109, 2006.

[40] R. M. Hirsch and J. R. Slack, "A nonparametric trend test for seasonal data with serial dependence," Water Resources Research, vol. 20, pp. 727-732, 1984.

[41] K. H. Hamed and A. Ramachandra Rao, "A modified Mann-Kendall trend test for auto correlated data," Journal of Hydrology, vol. 204, pp. 182-196, 1998.
[42] A. Araghi, M. Mousavi Baygi, J. Adamowski, J. Malard, D. Nalley, and S. M. Hasheminia, "Using wavelet transforms to estimate surface temperature trends and dominant periodicities in Iran based on gridded reanalysis data," Atmospheric Research, vol. 155, pp. 52-72, 2015.

[43] A. Sen, Poverty and Famines: An Essay on Entitlement and Deprivation, Clarendon Press, Oxford, UK, 1981.

[44] IMD, Rainfall and drought in India: A report. Indian Meteorological Department Government of India \& NCA, Agri Commission Report National Commission on Agriculture Ministry of Agriculture, GoI New Delhi, New Delhi, India, 1971.

[45] N. B. Guttman, "Comparing the palmer drought index and the standardized precipitation index," Journal of the American Water Resources Association, vol. 34, pp. 113-121, 1998.

[46] C. W. Thornthwaite, "An approach toward a rational classification of climate," Geographical Review, vol. 38, pp. 55-94, 1978.

[47] W. M. Alley, "The palmer drought severity index: limitations and assumptions," Journal of Applied Meterology and Climatology, vol. 23, no. 7, pp. 1100-1109, 1984.

[48] V. Krysanova, T. Vetter, and F. Hattermann, "Detection of change in drought frequency in the Elbe basin: comparison of three methods," Hydrological Sciences Journal, vol. 53, no. 3, pp. 519-537, 2008.

[49] M. P. Akhtar, L. B. Roy, and A. Sinha, A Comparative Study on Regional Drought Characterization Using Estimated Drought Indices in Conjunction with Trend Analysis in Peninsular India in Water Resources in Arid lands: Management and Sustainability, Springer International Publishing, Berlin, Germany, (In press), 2021.

[50] H. Peter and T. DeFoire, Final Report on Fit the Pearson Type III Distribution to Aviation Loads Data, US Department of Transportation Federal Aviation Administration, Washington, DC, USA, 2003.

[51] K. Subramanya, Engineering Hydrology, TMH, New Delhi India, 3rd edition, 2013.

[52] G. B. Pant and K. R. Kumar, Climates of South Asia, John Wiley, Hoboken, NJ, USA, 1997.

[53] T. B. McKee, N. J. Doesken, and J. Kleist, "Drought monitoring with multiple time scales," in Proceedings of the Ninth Conference on Applied Climatology, pp. 233-236, American Meteorology Society, Boston, MA, USA, January 1995.

[54] S. N. Rahmat, N. Jayasuria, and M. Bhuiyan, "Development of drought severity-duration-frequency curves in victoria Australia," Australian Journal of Water Resources, vol. 19, no. 1, pp. 31-42, 2015.

[55] B. Bonaccorso, A. Cancelliere, and G. Rossi, "An analytical formulation of return period of drought severity," Stochastic Environmental Research Risk Assessment, vol. 17, pp. 157-174, 2003.

[56] T. Raziei, B. Saghafian, A. Paulo, L. Pereira, and I. Bordi, "Spatial patterns and temporal variability of drought in western Iran," Water Resources Management, vol. 23, pp. 439-455, 2009.

[57] M. Hayes, D. A. Wood, and M. Svoboda, Standardized Precipitation Index User Guide, World Meteorological Organization, Geneva, Switzerland, 2012.

[58] A. Ramachandra Rao and G. Padmanabhan, "Analysis and modeling of palmer's drought index series," Journal of $\mathrm{Hy}$ drology, vol. 68, pp. 211-229, 1984.

[59] J. Zhai, B. Su, V. Krysanova, T. Vetter, C. Gao, and T. Jiang, "Spatial variation and trends in PDSI and SPI indices and their 
relation to streamflow in 10 large regions of China," Journal of Climate, vol. 23, pp. 649-663, 2009.

[60] Y. Yihdego, B. Vaheddoost, and R. A. Al-Weshah, "Drought indices and indicators revisited," Arabian Journal of Geosciences, vol. 12, p. 69, 2019.

[61] M. A. Niaz, M. A. Faiz, and W. Yongxia, "Development of an integrated weighted drought index and its application for agricultural drought monitoring," Arabian Journal of Geosciences, vol. 14, no. 6, pp. 1-12, 2021. 